\title{
Circadian regulation of mitochondrial uncoupling and lifespan
}

\author{
Matt Ulgherait', Anna Chen (10 2, Sophie F. McAllister², Han X. Kim', Rebecca Delventhal (1) 1, \\ Charlotte R. Wayne ${ }^{3}$, Christian J. Garcia ${ }^{4}$, Yocelyn Recinos ${ }^{5}$, Miles Oliva ${ }^{2}$, Julie C. Canman 6 , Martin Picard ${ }^{7}$, \\ Edward Owusu-Ansah ${ }^{4} \&$ Mimi Shirasu-Hiza (i) ${ }^{1 凶}$
}

Because old age is associated with defects in circadian rhythm, loss of circadian regulation is thought to be pathogenic and contribute to mortality. We show instead that loss of specific circadian clock components Period (Per) and Timeless (Tim) in male Drosophila significantly extends lifespan. This lifespan extension is not mediated by canonical diet-restriction longevity pathways but is due to altered cellular respiration via increased mitochondrial uncoupling. Lifespan extension of per mutants depends on mitochondrial uncoupling in the intestine. Moreover, upregulated uncoupling protein UCP4C in intestinal stem cells and enteroblasts is sufficient to extend lifespan and preserve proliferative homeostasis in the gut with age. Consistent with inducing a metabolic state that prevents overproliferation, mitochondrial uncoupling drugs also extend lifespan and inhibit intestinal stem cell overproliferation due to aging or even tumorigenesis. These results demonstrate that circadianregulated intestinal mitochondrial uncoupling controls longevity in Drosophila and suggest a new potential anti-aging therapeutic target.

\footnotetext{
${ }^{1}$ Department of Genetics and Development, Columbia University Vagelos College of Physicians and Surgeons, New York, NY 10032, USA. ${ }^{2}$ Columbia College, New York, NY 10027, USA. ${ }^{3}$ Department of Neurology, Columbia University Vagelos College of Physicians and Surgeons, New York, NY 10032, USA. ${ }^{4}$ Department of Physiology and Cellular Biophysics, Columbia University Vagelos College of Physicians and Surgeons, New York, NY 10032, USA. ${ }^{5}$ Department of Systems Biology, Columbia University Vagelos College of Physicians and Surgeons, New York, NY 10032, USA. ${ }^{6}$ Department of Pathology and Cell Biology, Columbia University Vagelos College of Physicians and Surgeons, New York, NY 10032, USA. 7 Departments of Psychiatry and Neurology,

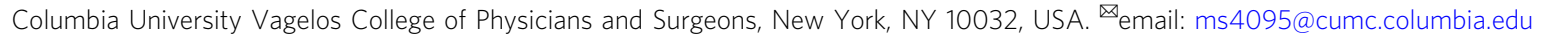


M

ost animals exhibit behavior and physiologies linked to a 24-h circadian rhythm, controlled by endogenous circadian clocks. These clocks are composed of transcriptional feedback loops regulating the expression of genes controlling diverse cellular functions. In Drosophila, the circadian transcriptional activators Clock and Cycle induce the oscillating expression of hundreds of target genes, including period (per) and timeless (tim), which encode repressors of Clock and Cycle activity (Fig. 1a). Because organisms lose circadian rhythmicity with age, it has been hypothesized that loss of circadian regulation contributes to aging and limits lifespan. Specifically, there has been interest in the impact of circadian-regulated metabolism on
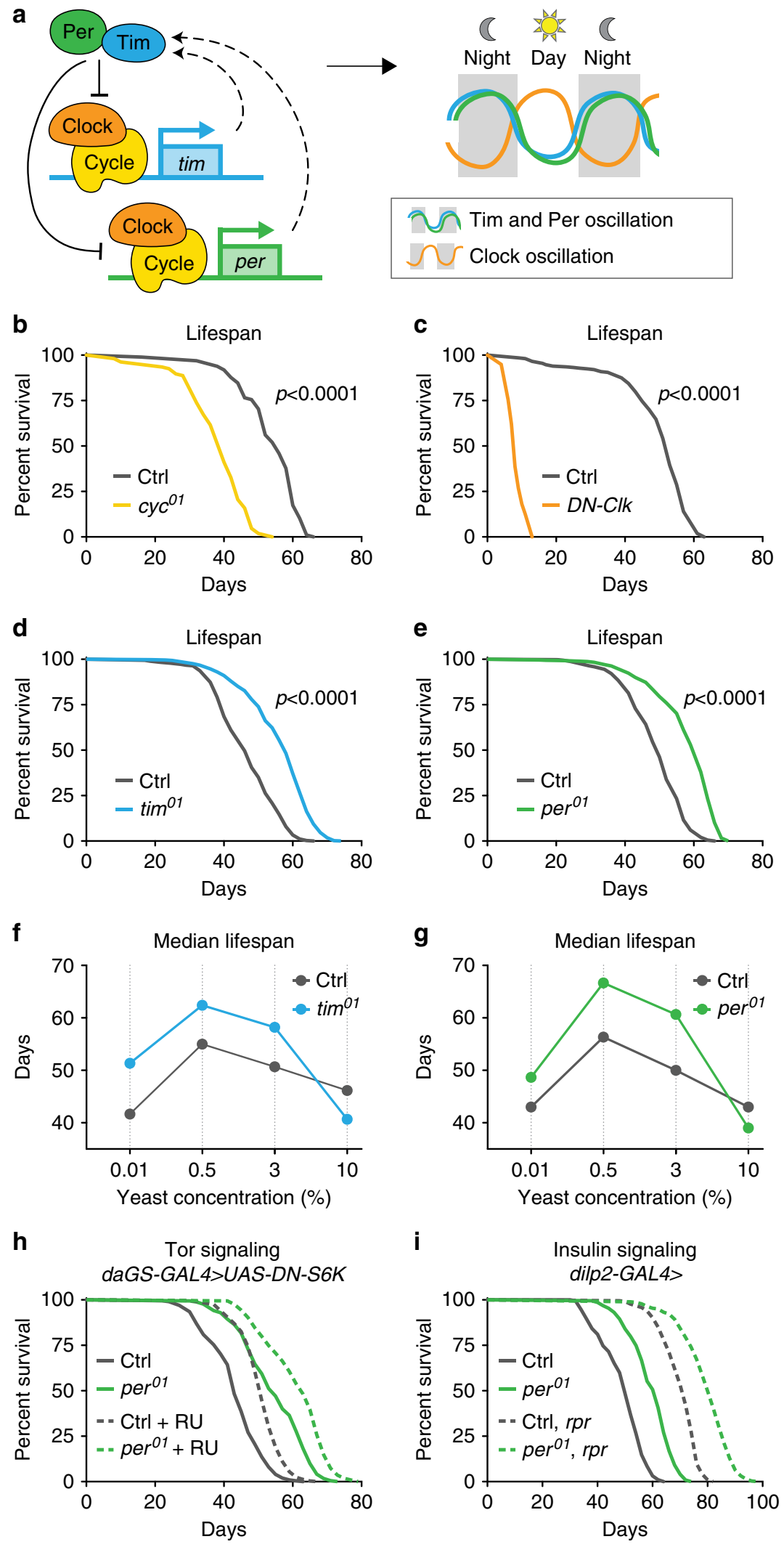
Fig. 1 Loss of the repressive arm of the transcriptional circadian clock extends male lifespan. a Schematic of core molecular clock components and circadian transcriptional feedback loop. Relative to controls (gray), mutant males lacking Cyc function (yellow, b) or expressing dominant-negative clock (daGS > UAS-DN-Clock flies fed RU486, orange, c) show reduced lifespan ( $p<0.0001$ for each circadian mutant vs. control). In contrast, tim 01 mutants (blue, d) and per ${ }^{01}$ mutants (green, e) live longer than controls (gray), even on diets with different concentrations of yeast extract (f, $\mathbf{g}$ ), except for the highest yeast diet ( $p<0.001$ for each circadian mutant vs. control on each diet). $\mathbf{h}$ per ${ }^{01}$ mutants with daGS $>$ UAS-DN-S6K fed either RU486 (dashed lines) or vehicle (solid lines) exhibited similar lifespan extension relative to controls containing daGS $>U A S-D N-S 6 K$. i per ${ }^{01}$ mutant males containing either the dilp2-GAL4 driver alone (solid lines) or dilp2-GAL4 > UAS-reaper and ablated for insulin-producing cells (dashed lines) exhibit similar lifespan extension relative to controls. See Supplementary Table 1 for $n$ and $p$ values for lifespan experiments, particularly multicurve comparisons; $p$ values were obtained by log-rank analysis.

lifespan and aging, as many mechanisms regulating organismal lifespan involve large metabolic changes, including circadianregulated metabolic genes ${ }^{1,2}$. Most reports investigating core molecular clock components and lifespan examined the circadian transcriptional activators (i.e., Clock or Cycle) and found that disruption of these activators led to metabolic dysfunction and shortened lifespan ${ }^{3-7}$. Studies examining loss of circadian transcriptional repressors (i.e. Period, Timeless, or Cryptochrome) and the impact on metabolism, healthspan, and lifespan have been more controversial ${ }^{5,8-12}$. While a number of studies identified the detrimental effects of loss of the transcriptional repressor per 2 in the mouse ${ }^{13,14}$ or per in Drosophila $9,15,16$, loss of per2 may also have beneficial effects on specific aspects of healthspan and lifespan ${ }^{12,17-19}$. For example, loss of per2 in the whole mouse has been associated with increased metabolic rate, lowered fat storage, increased leptin levels, and decreased insulin resistance compared with control animals, indicating a possibly favorable metabolic state under ad libitum feeding conditions ${ }^{17,20}$. Thus, while loss of circadian regulation has pathological effects, loss of circadian regulation in specific genetic and environmental contexts may have metabolic advantages. The specific mechanisms underlying circadian-regulated metabolism and their roles in aging and longevity remain unclear.

Here, we show that loss of the repressive arm of the core circadian clock extends male Drosophila lifespan. Loss of period also induces a highly active metabolic state characterized by increased mitochondrial uncoupling; this lifespan extension is due to upregulation of the endogenous mitochondrial uncoupling protein (UCP) UCP4C, specifically in the intestine. Loss of per or upregulation of UCP4C attenuates age-related decline in gut homeostasis. These genetic phenotypes, including longevity extension and improved gut homeostasis, are recapitulated by feeding Drosophila low doses of mitochondrial uncoupling drugs.

\section{Results}

Loss of tim or per extends lifespan in male Drosophila. To investigate how loss of specific circadian regulators influence aging, we examined the lifespans of four established arrhythmic Drosophila mutants (Fig. 1a): three genomic mutants, cycle $\left(c y c^{01}\right)$, period $\left(\right.$ per $\left.^{01}\right)$, and timeless $\left(\right.$ tim $\left.^{01}\right)$, and flies ubiquitously expressing a dominant-negative form of Clock $(D N-C l o c k)^{21}$. Consistent with other reports ${ }^{3-6}$, functional disruption of the circadian transcriptional activators Cycle or Clock shortened the lifespan of male flies relative to controls (Fig. 1b, c). In contrast, functional disruption of the circadian transcriptional repressors Per and Tim significantly increased lifespan (15-20\%) relative to controls; this appeared to be a male-specific effect (Fig. 1d, e). To confirm that lifespan extension was due to the loss of Per protein, we restored Per expression using the UAS-GAL4 system ${ }^{22}$. Expressing either of the two independent period transgenes using either the timeless-GAL4 driver or the ubiquitin-GAL4 driver in the per ${ }^{01}$ null background reverted per mutant lifespan to that of control animals (Supplementary Fig. 1A, B). Thus, loss of Per expression extends lifespan.
The classic method of lifespan extension is dietary restriction (DR). We showed previously that per ${ }^{01}$ and $t^{2} \mathrm{~m}^{01}$ mutants are not diet-restricted-that is, these mutants eat more, not less, than controls (ref. ${ }^{19}$; see also Fig. 2a). However, loss of Per and Tim might mimic physiological changes associated with DR. If so, DR should not further extend the lifespan of male $\mathrm{per}^{01}$ and $\mathrm{tim}^{01}$ mutants. In Drosophila, DR-mediated lifespan extension is accomplished by titration of protein (yeast extract (YE)). To test response to DR, we fed per $^{01}$ and tim ${ }^{01}$ null mutants and controls four different concentrations of YE: 0.01\% (low), 0.5\% (DR), 3\% (standard), and 10\% (high). As we showed previously for female per $^{01}$ and tim $^{01}$ mutants ${ }^{10}$, male per 01 and tim $^{01}$ mutants exhibited DR-induced lifespan extensions similar to controls and lived longer than controls on most dietary protein concentrations (Fig. 1f, g). The lifespan of these circadian mutants was similar to that of control animals only at very high yeast concentrations (10\%), which shortens lifespan. Thus, the extended longevity of per $^{01}$ mutants appears to be independent of DR.

We next tested if per $^{01}$ mutants exhibit canonical changes in DR-associated mechanisms of longevity, including: decreased insulin signaling, measured by decreased phosphorylation of Akt protein; decreased TOR signaling, measured by decreased phosphorylation of S6K; and increased autophagy, measured by increased lipidation of Atg8. Surprisingly, per ${ }^{01}$ males were atypical for these hallmarks of longevity and, at some points in the circadian cycle, exhibited the opposite phenotype as predicted for long-lived mutants (Supplementary Fig. 1C). We further used genetic manipulations to determine if the inhibition of TOR signaling or the inhibition of insulin-like signaling (ILS) is responsible for the longevity of $\mathrm{per}^{01}$ mutants. To test the inhibition of TOR signaling, we ubiquitously suppressed TORC1 signaling in adulthood by inducible (RU486-mediated) overexpression of a dominant-negative form of the downstream kinase S6K, which is known to extend lifespan ${ }^{23}$. The inhibition of TORC1 signaling extended the lifespans of both control animals and per ${ }^{01}$ mutants to a similar magnitude (Fig. 1h), suggesting that the longevity of per ${ }^{01}$ mutants is independent of TORC1 inhibition. Feeding RU486 to either controls or per ${ }^{01}$ mutants lacking the UAS transgene had no influence on lifespan (Supplementary Fig. 1D). To test the inhibition of ILS, we performed partial genetic ablation of insulin-producing cells in the fly brain using the proapoptotic gene reaper ${ }^{24}$. This extended the lifespans of both control animals and per $^{01}$ mutants to a similar magnitude (Fig. 1i), suggesting that per $^{01}$-associated lifespan extension is independent of insulin-like signaling inhibition. Together, these results suggest that the longevity phenotype of per $^{01}$ males is not due to canonical longevity mechanisms but due to a different, independent pathway.

per mutants exhibit a high metabolic rate. As metabolism and lifespan are linked, we set out to investigate the metabolism of long-lived per ${ }^{11}$ mutants. As shown in our previous work ${ }^{19}$, per $^{01}$ males exhibited hyperphagia (increased feeding), decreased 
a

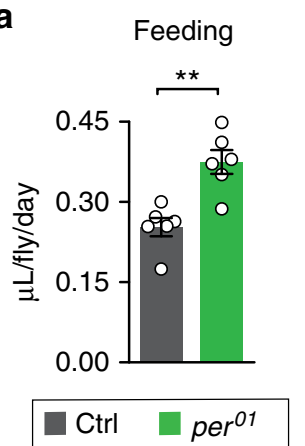

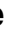

e

Respiration rate (whole fly)

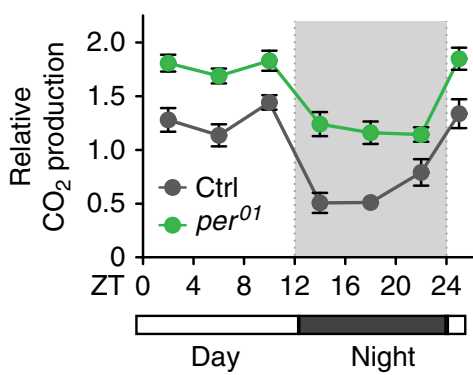

g Oxygen consumption

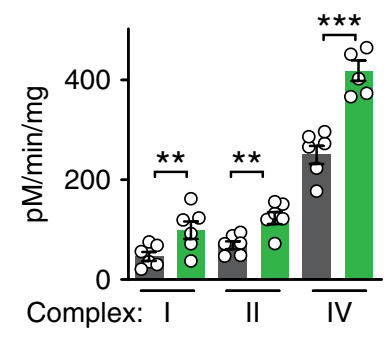

b

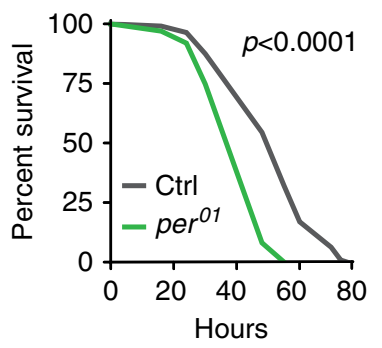

c

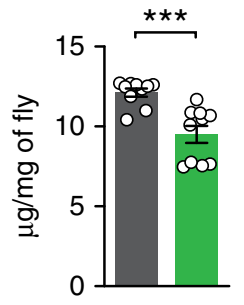

TAG

starvation d Respiration rate

daGS>UAS-per
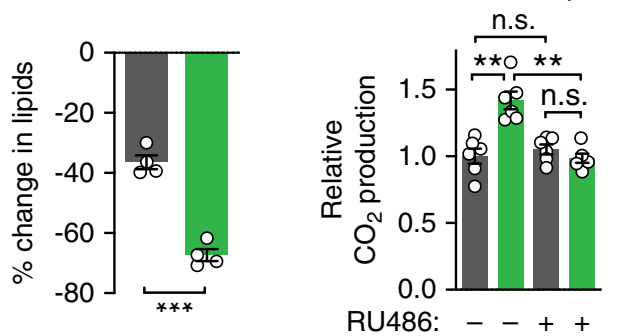

f

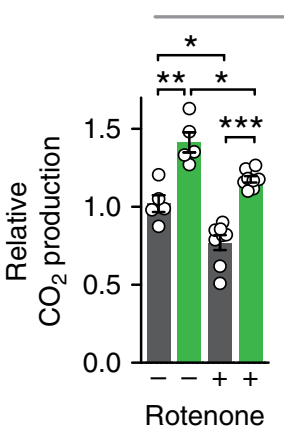

h

OCR leak respiration

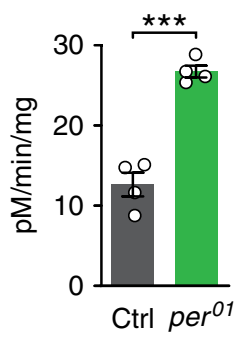

i

Mitochondrial memb. potential (JC-1)

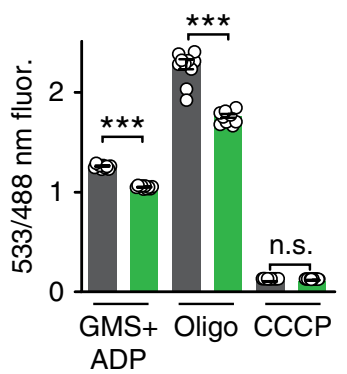

Respiration rate (whole fly)
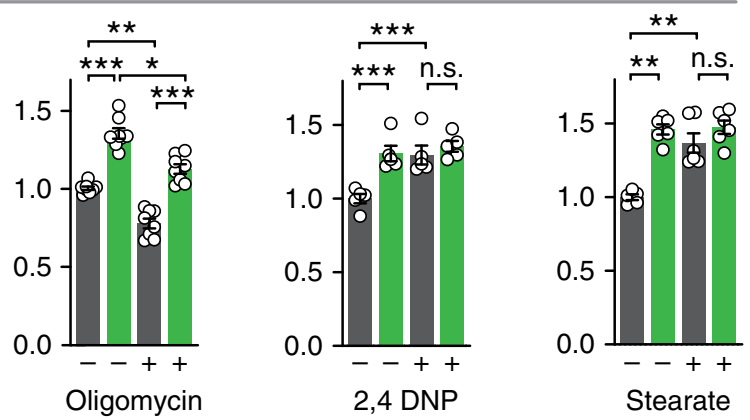

j

Cold shock recovery

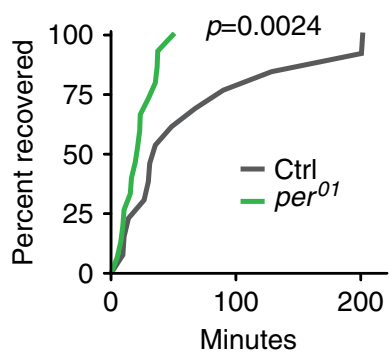

Fig. 2 period mutants exhibit high metabolic rate due to mitochondrial uncoupling. Relative to controls (gray), per01 mutants (green) exhibited: a increased feeding rate ( $n=6$ vials of ten flies/condition, $p<0.01)$; b decreased survival upon starvation $(n \geq 99$ flies per condition, $p<0.001)$; c lower baseline levels of lipids (left) and increased rate of lipid utilization after $24 \mathrm{~h}$ of starvation (right), as shown by quantification of triacylglyceride (TAG) levels ( $n \geq 4$ samples/condition, 5 flies/sample, both $p<0.0001$ ); $\mathbf{d}$ increased respiration, which was reverted by ubiquitous overexpression of Per during adulthood ( $n=6$ groups of 10 flies per condition); e higher $\mathrm{CO}_{2}$ production over the circadian day ( $n=6$ groups of 10 flies/condition and timepoint); $\mathbf{f}$ higher respiration rates after $24 \mathrm{~h}$ of feeding with rotenone and oligomycin, but not with 2,4-DNP, or stearic acid ( $n \geq 5$ groups of 10 flies/condition); $\mathbf{g}$ increased oxygen consumption rate when stimulated through complexes I, II, and IV relative to controls ( $n=4-6$ oxygraph runs per condition); $\mathbf{h}$ increased leak respiration, using high-resolution respirometry on purified mitochondria ( $n=5$ oxygraph runs per condition, $p<0.001)$; i lower membrane potential, measured by JC- 1 staining of purified mitochondria $(n=10$ mitochondrial preps per condition, $p<0.001)$; and $\mathbf{j}$ faster recovery from cold shock $(n=26-30$ flies per condition, $p<0.001$ ). See Supplementary Information for $n$ if not listed here; ${ }^{n \cdot s} p>0.05,{ }^{\star} p<0.05,{ }^{\star \star} p<0.01,{ }^{\star \star \star} p<0.001 ; p$ values were obtained by unpaired two-tailed $t$-test $(\mathbf{a}, \mathbf{c}, \mathbf{e}, \mathbf{g}$-i), ANOVA followed by Tukey's post-hoc test $(\mathbf{d}, \mathbf{f})$, and log-rank analysis (b, j); error bars represent SEM.

starvation resistance, low levels of lipid storage, and increased starvation-induced lipid utilization relative to controls (Fig. 2a-c, Supplementary Fig. 2A). Because per ${ }^{01}$ mutants eat more but are leaner than controls, we tested for hyperactive metabolic rate by measuring $\mathrm{CO}_{2}$ production. Consistent with our previous characterization, per ${ }^{01}$ mutants produced more $\mathrm{CO}_{2}$ throughout the circadian cycle relative to controls, which was reverted by exogenous Per expression (Fig. 2d, e); respiration rate was not affected in RU486 feeding controls (Supplementary Fig. 2B). Respiration rate is significantly affected by the mitochondrial function of oxidative phosphorylation. To determine if increased oxidative phosphorylation caused this increased metabolic output, flies were fed sublethal doses of mitochondrial complex inhibitors: rotenone, which blocks complex I of the mitochondrial electron transport chain (ETC), or oligomycin, which blocks complex $\mathrm{V}$, the $\mathrm{F}_{0} \mathrm{~F}_{1}$ ATP synthase. While these compounds inhibited the $\mathrm{CO}_{2}$ output of both control and per ${ }^{01}$ animals to a similar degree, $p e r^{01}$ flies still had higher respiration rates than controls (Fig. 2f). This suggests that their increased respiration is independent of mitochondrial ATP synthesis and instead implicates a different mitochondrial function, such as mitochondrial uncoupling.

Mitochondrial uncoupling increases respiration by dissipation of the proton gradient and uncoupling of oxidative 
phosphorylation from ATP synthesis, creating futile cycles of respiration and generating heat, as in mammalian brown fat. To test the effects of mitochondrial uncoupling on respiration, we fed per $^{01}$ mutants and control flies two different mitochondrial uncoupling compounds: 2,4-dinitrophenol (2,4-DNP), a proton ionophore that dissipates the proton gradient; and stearic acid, which induces mitochondrial uncoupling by activating endogenous UCP activity. Treatment with either drug increased the respiration of control flies to levels similar to those of per $^{01}$ mutants but did not increase the respiration of per ${ }^{01}$ mutants (Fig. 2f). These results suggest that the increased respiration of per ${ }^{01}$ mutants is due to increased mitochondrial uncoupling and that per $^{01}$ mutants may already be maximally uncoupled within viable parameters. Higher doses of uncoupling drugs were lethal to both genotypes.

To directly test whether per ${ }^{01}$ mutants were mitochondrially uncoupled relative to controls, we assessed mitochondrial function in vitro. We purified mitochondria from per $^{01}$ mutants and controls and measured $\mathrm{O}_{2}$ consumption. per ${ }^{01}$ mutant mitochondria exhibited increased $\mathrm{O}_{2}$ consumption when initiated through ETC complexes I, II, and IV (Fig. 2g). This increased $\mathrm{O}_{2}$ consumption was not due to increased mitochondrial ETC protein abundance or increased enzymatic activity (Supplementary Fig. 2C, D). Instead, per ${ }^{01}$ mitochondria exhibited two critical hallmarks of mitochondrial uncoupling relative to control mitochondria: increased leak respiration, measured by higher oxygen consumption after oligomycin treatment (Fig. 2h); and decreased membrane potential, or disrupted proton gradient, measured by the membrane potential dye sensor JC-1, both during steady-state ATP generation and after inhibition by oligomycin (Fig. 2i). Finally, to assess heat generation, another hallmark of mitochondrial uncoupling, we performed cold shock recovery assays on per ${ }^{01}$ mutants and control animals (Fig. 2j). After $1 \mathrm{~h}$ of cold shock at $4^{\circ} \mathrm{C}$, per ${ }^{01}$ mutants recovered significantly faster than controls, suggesting that per ${ }^{01}$ mutants may generate more heat than controls. Thus, loss of Per protein increases mitochondrial uncoupling.

Uncoupling protein UCP4C is required for per mutant longevity. To determine if this increased mitochondrial uncoupling is required for the lifespan extension of per ${ }^{01}$ mutants, we genetically manipulated the expression of endogenous proteins that cause mitochondrial uncoupling. Like many animals, Drosophila can undergo mitochondrial uncoupling by induction of UCPs, including UCP $4 A, B$, and $C^{25,26}$. Of these, we found that the expression of $U c p 4 B$ and $U c p 4 C$ is circadian-regulated in control flies and constitutively high in per $^{01}$ mutants (Fig. $3 a-c$ ). To test the role of UCPs in the metabolic phenotype observed in per ${ }^{01}$ mutants, we first disrupted the expression of both UCP4B/ $\mathrm{C}$ proteins using a mutant containing a piggyBac transposon in the intergenic region between these two closely linked Ucp4 genes (Supplementary Fig. 3A, B). Next, to determine if period nulls exhibit true mitochondrial uncoupling via classic mitochondrial UCPs, we tested stimulation of mitochondrial respiration in the presence of palmitate and reversal by the UCP inhibitor guanosine nucleotide (GTP) suppression of respiration. Palmitate and GTP are respectively known to stimulate and suppress classic mitochondrial UCPs ${ }^{27-29}$. Indeed, per ${ }^{01}$ flies showed significantly increased respiration in the presence of palmitate, which was reversed by the addition of GTP (Supplementary Fig. 3C). per ${ }^{01}$ flies with disrupted UCP4B/C expression showed no response to palmitate or GTP indicating that the uncoupled respiration is due to UCP gene function. In addition, disruption of $\mathrm{UCP} 4 \mathrm{~B} / \mathrm{C}$ in per $^{01}$ flies reverted other hallmarks of uncoupling: mitochondrial leak respiration
(Fig. 3d); mitochondrial membrane potential (Fig. 3e); mitochondrial oxygen consumption rate (Supplementary Fig. 3D); and whole-animal cold shock recovery rates (Fig. 3f). Disruption of the UCP4B/C expression not only reverted mitochondrial uncoupling but also reverted per $^{01}$ lifespan to that of controls, suggesting that mitochondrial uncoupling causes lifespan extension (Fig. 3g). To inhibit UCPs by an orthogonal mechanism, we performed RNAi-mediated knockdown of UCP4A, B, and C in adulthood throughout the whole body in both per $^{01}$ mutants and controls. Consistent with our mutant analysis, knockdown of UCP4B or UCP4C, but not UCP4A, reverted per $^{01}$ lifespan to that of control animals (Supplementary Fig. 3E-G). Thus, the Ucp $4 \mathrm{~B} / \mathrm{C}$ expression is necessary for the longevity and metabolic phenotypes of per $^{01}$ mutants.

Finally, to determine if increased UCP expression is sufficient to extend the lifespan of WT animals, we ubiquitously overexpressed UCP4C at low levels during adulthood using conditional GeneSwitch drivers induced by the drug RU486. Flies overexpressing UCP4C showed mitochondrial uncoupling phenotypes very similar to per $^{01}$ mutants; increased mitochondrial leak respiration (Fig. 3h), decreased mitochondrial membrane potential (Fig. 3i), increased $\mathrm{CO}_{2}$ production (Supplementary Fig. 4A), and faster cold shock recovery (Supplementary Fig. 4B). Most importantly, the constitutive expression of UCP4C extended the lifespan of otherwise wild-type flies to the same extent as per $^{01}$ mutants, with no effect on per $^{01}$ mutants (Fig. 3j-k). Feeding these low doses of RU486 alone had no effect on either metabolic or lifespan phenotypes when flies lacked the UAS transgene (Supplementary Fig. 3C-G). These results suggest that UCP4C functions in the same pathway as Per to extend per ${ }^{01}$ mutant lifespan and that increased expression of the mitochondrial uncoupling protein UCP4C extends lifespan. Taken together, our data point to mitochondrial uncoupling as the major circadian-regulated physiology directly responsible for the extended lifespan of per $^{01}$ mutants.

Lifespan extension is mediated by loss of Per in intestines. While circadian sleep/wake cycles are coordinated by a neuronal master clock in the brain, many other circadian functions are regulated by peripheral clocks in specific tissues ${ }^{30}$. To determine whether the master clock or a peripheral circadian clock mediates per $^{01}$ longevity, we rescued period expression in different organ systems via the UAS-GAL4 system. While ubiquitous expression of Per protein during adulthood reverted the lifespan of $p^{01}$ mutants to that of controls (Fig. 4a), neuronal expression of Per did not (Fig. 4b). In contrast, intestinal expression of Per in the whole intestine or specifically in intestinal stem cells (ISCs) and enteroblasts (EBs) reverted per $^{01}$ lifespan to that of controls (Fig. 4c-e). This result suggests that loss of Per in the intestine is required for lifespan extension of per ${ }^{01}$ mutants.

To directly test if loss of per in the intestine is sufficient to extend lifespan, we used a UAS-GAL4-based CRISPR system to disrupt the period gene ${ }^{31,32}$. Ubiquitous deletion of per via the daughterless GeneSwitch driver (daGS-Gal4) was sufficient to extend lifespan of control flies but not per ${ }^{01}$ mutants (Fig. 4f). Similar to per ${ }^{01}$ mutants, flies with ubiquitous disruption of period were completely arrhythmic in constant darkness (Supplementary Fig. 5A). We then performed tissue-specific CRISPRmediated disruption of period in either the whole intestine or specifically in ISCs/EBs during either development or adulthood and found that any of these manipulations was sufficient to extend lifespan (Fig. 4g, h). Disruption of period in the whole intestine or ISCs and EBs had no influence on circadian locomotor activity (Supplementary Fig. 5B, C). To control for CRISPR-mediated DNA damage, we disrupted an unrelated gene 
a

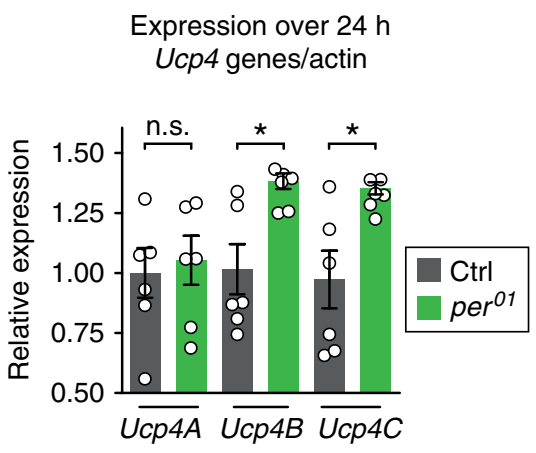

d

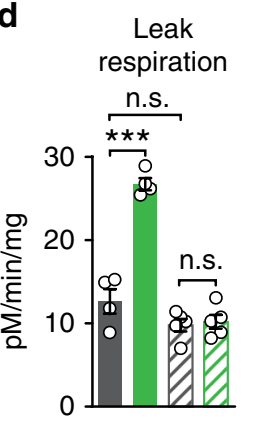

b

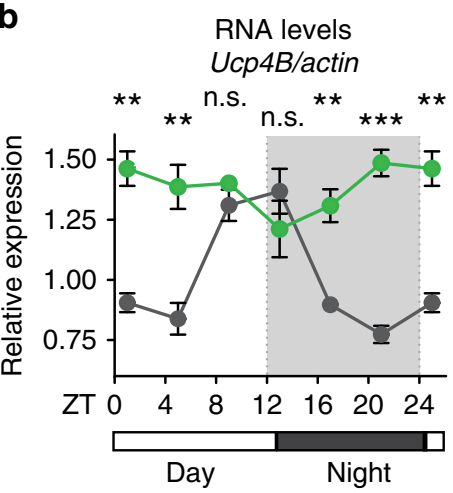

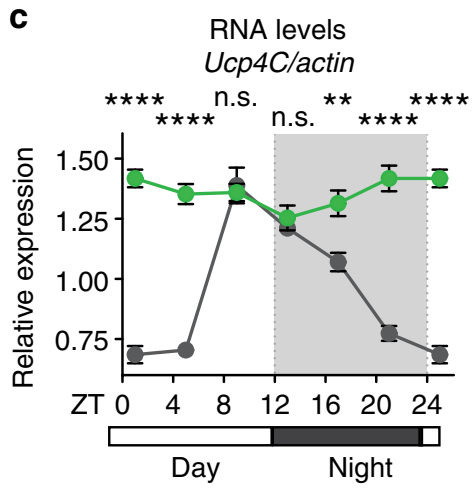

f

Cold shock recovery memb. potential (JC-1)
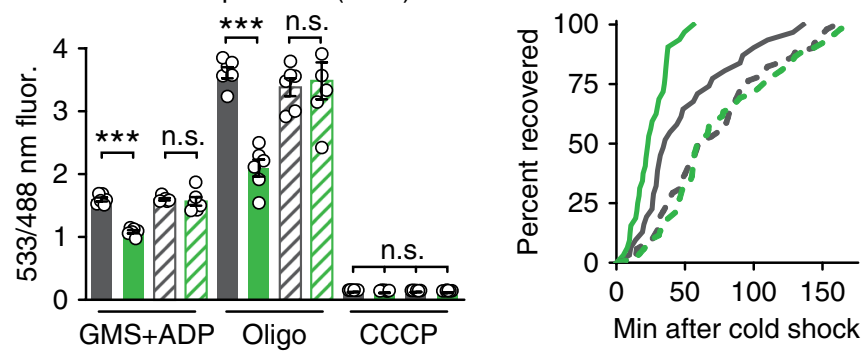

g

Lifespan

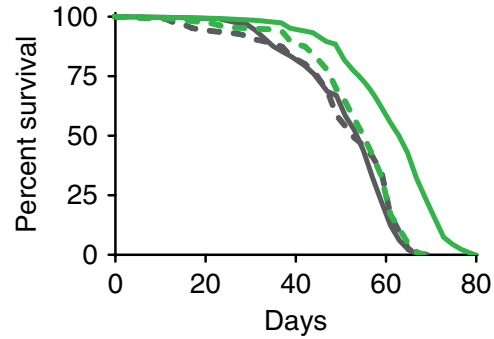

Ctrl $\square \mathrm{Ctrl} ; p B-U c p 4 B / C$

$\operatorname{per}^{01} \square p e r^{01} ; p B-U c p B / C$

$$
\begin{aligned}
& \text { - Ctrl } \quad \text { - Ctrl; } p B-U c p 4 B / C \\
& -\operatorname{per}^{01} \ldots \operatorname{per}^{01} ; p B-U c p B / C
\end{aligned}
$$

h<smiles>[13CH][13CH3]</smiles>
i men me

Mitochondrial

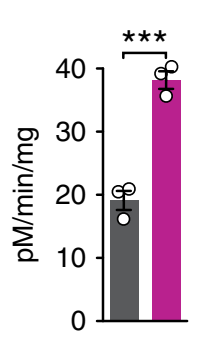

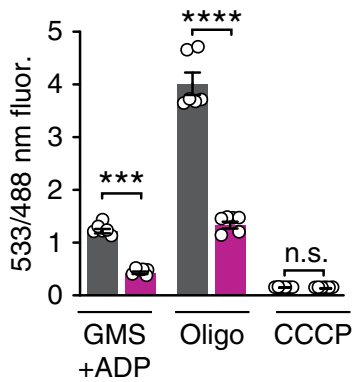

\section{DaGS-GAL4>UAS-UcP4C}

口- RU

$+\mathrm{RU}$

j

Induced mito. uncoupling daGS-GAL4> UAS-Ucp $4 C$

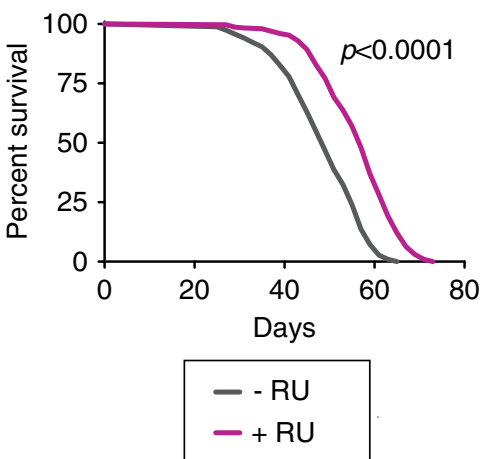

k Induced mito. uncoupling ubiquitin-GAL4> UAS-Ucp $4 C$

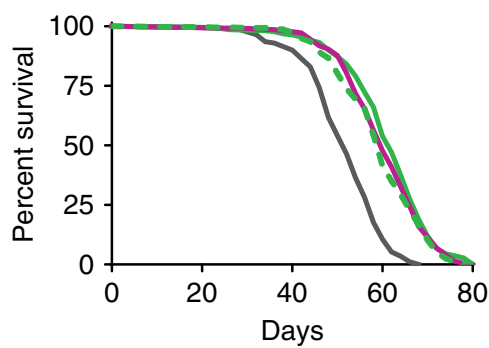

$$
\begin{array}{ll}
\text { - Ctrl } & \text { - Ctrl; UAS-Ucp } 4 C \\
\text { - per } & \text { - - per } \\
& 01 ; \text { UAS-Ucp4C }
\end{array}
$$

Fig. 3 UCP4C is necessary for period mutant lifespan and sufficient to extend wild-type lifespan. Relative to controls ( $\mathrm{gray}$ ), per $\mathrm{p}^{\mathrm{1}} \mathrm{mutants}$ ( $\mathrm{green}$ ) exhibited: a higher expression of $U c p 4 B$ and $U c p 4 C$ but not $U c p 4 A$; and constitutively high expression of $\mathbf{b} U c p 4 B$ and $\mathbf{c} U c p 4 C$, both of which are circadianregulated in controls. Relative to controls (gray), per ${ }^{01}$ mutants (green) also exhibited the following phenotypes, which were reverted by suppression of $U c p 4 B / C$ expression, comparing flies with (dashed lines) or without (solid lines) piggyback mutation of Ucp4B/C: $\mathbf{d}$ increased leak respiration by purified mitochondria; e decreased mitochondrial membrane potential; f faster cold shock recovery; and $\mathbf{g}$ increased lifespan. Relative to vehicle-fed controls (gray), daGS > UAS-Ucp4C flies fed RU486 to induce constitutive UCP4C overexpression (magenta) exhibited: $\mathbf{h}$ higher leak respiration ( $p<0.001$ ); $\mathbf{i}$ lower mitochondrial membrane potential $(p<0.001)$; and $\mathbf{j}$ increased lifespan $(p<0.0001)$. $\mathbf{k}$ Ubiquitous overexpression of UCP4C in otherwise wild-type flies was sufficient to extend lifespan (gray, dashed) relative to driver-only controls (gray, solid). In per ${ }^{01}$ mutants (green, solid), overexpression of UCP4C did not further extend the lifespan of per ${ }^{01}$ mutants (green, dashed). See Supplementary Table 1 for $n$ and statistical analysis of lifespans, particularly multicurve comparisons; ${ }^{n . s .} p>0.05,{ }^{\star} p<0.05,{ }^{* \star} p<0.01,{ }^{\star \star \star} p<0.001,{ }^{\star \star \star \star *} p<0.001 ; p$ values were obtained by unpaired two-tailed $t$-test $(\mathbf{a}, \mathbf{h}, \mathbf{i})$, ANOVA followed by Tukey's post-hoc test (d, e), and log-rank analysis $(\mathbf{f}, \mathbf{g}, \mathbf{j}, \mathbf{k})$; error bars represent SEM.

involved in sperm storage in females $(\operatorname{acp} 98 A B)^{33}$ using the same inducible GAL4 drivers. $a c p 98 A B$ disruption did not extend lifespan using any of these tissue-specific drivers (Supplementary Fig. 5D-F). Similarly, RU486 feeding had no influence on control and per ${ }^{01}$ mutants lacking UAS transgenes (Supplementary
Fig. 5G-I). CRISPR-targeted disruption of period in ISCs/EBs resulted in $>90 \%$ reduction in per transcript in the intestine after 30 days of induction (Supplementary Fig. 5J). Taken together, these data show that the intestinal circadian clock plays a role in limiting lifespan in Drosophila. 
Tissue-specific rescue of per ${ }^{01}$ mutants
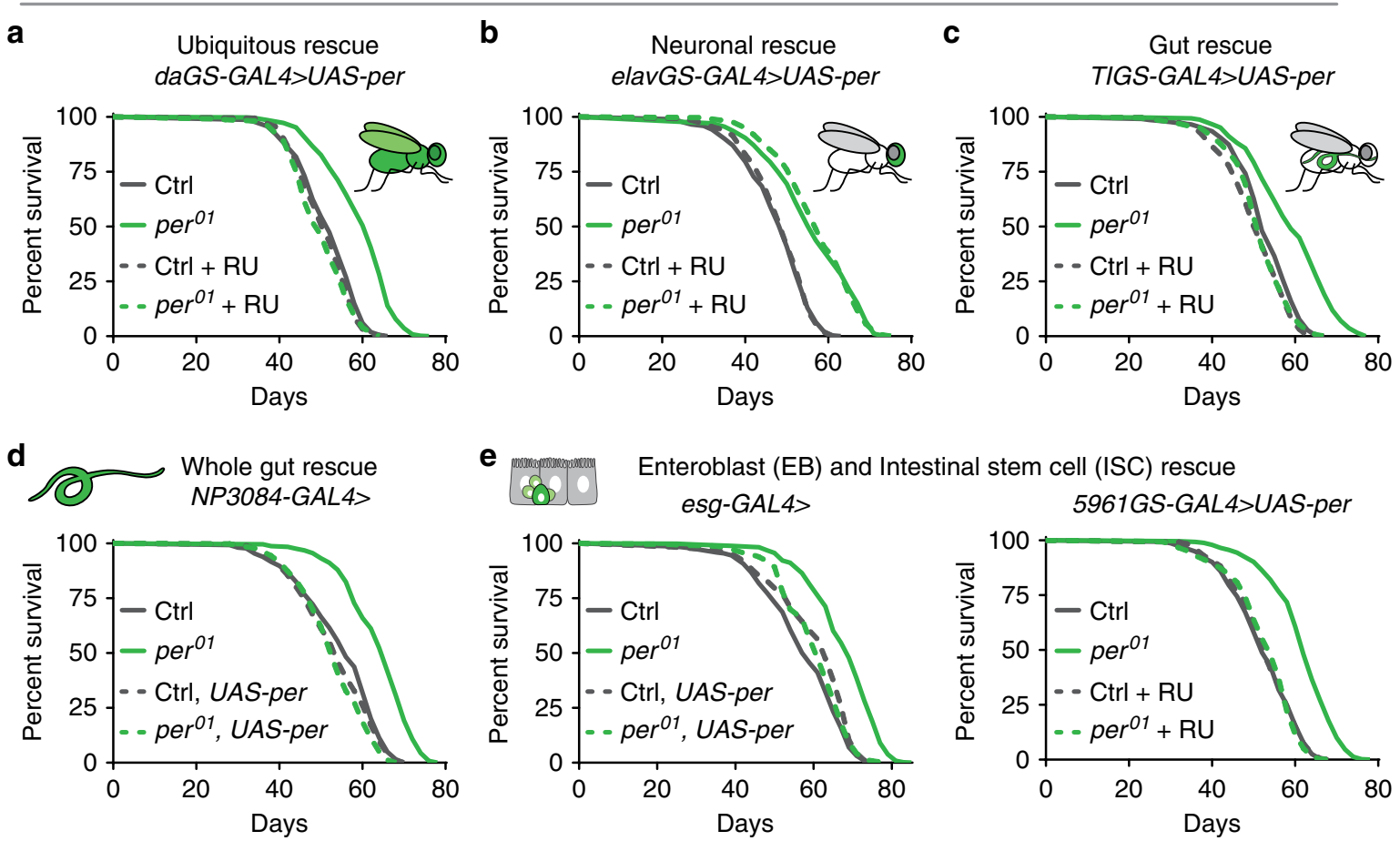

Tissue-specific CRISPR-mediated disruption of period
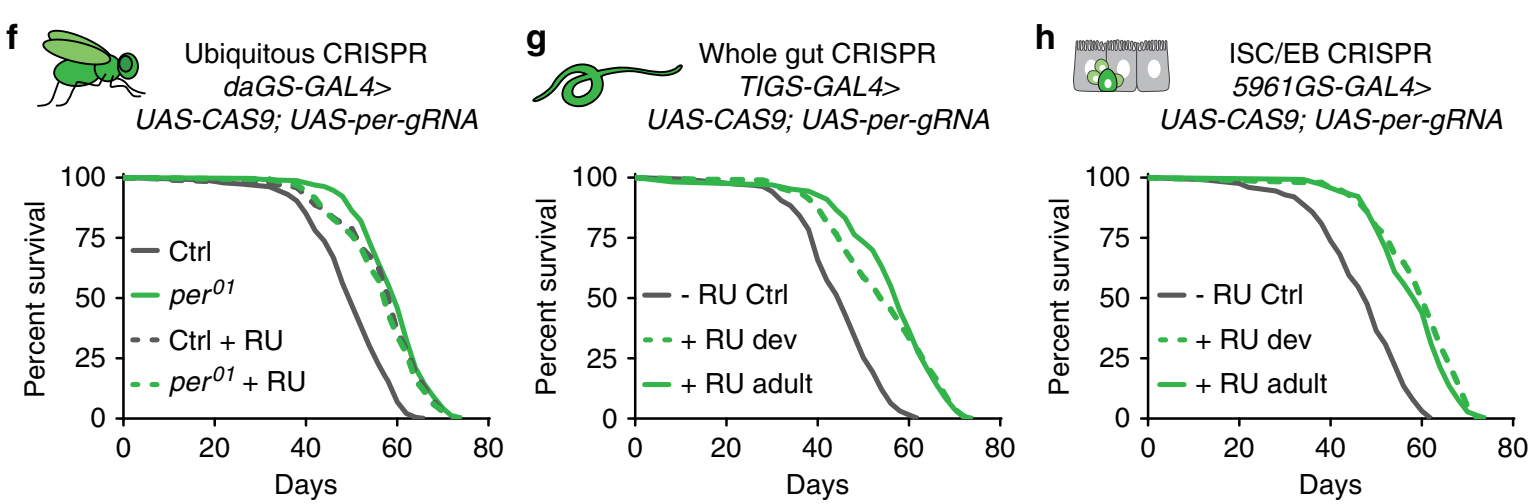

Fig. 4 Lifespan extension is mediated by loss of Per specifically in the intestine. Tissue-specific rescue of Per expression (dashed lines) in the per 01 background (green) and controls (gray). a Ubiquitous rescue of Per during adulthood was sufficient to revert per01 lifespan to control levels. While neuronal overexpression of $\operatorname{Per}(\mathbf{b})$ during adulthood did not revert per ${ }^{01}$ lifespan, intestinal overexpression of Per (c, d) was sufficient to revert per ${ }^{01}$ lifespan to control levels. e Rescue of Per specifically in intestinal stem cells (ISCs) and enteroblasts (EBs) during development or adulthood reverted per01 lifespan to control levels. $\mathbf{f}$ Loss of period through ubiquitous CRISPR-mediated deletion during adulthood extended lifespan of otherwise wild-type flies, with no further lifespan extension in per ${ }^{01}$ nulls. CRISPR-mediated deletion of period in $\mathbf{g}$ the intestine or $\mathbf{h}$ IScs and EBs also extended lifespan. See Supplementary Table 1 for $n$ and $p$ values for lifespan experiments, particularly multicurve comparisons; $p$ values were obtained by log-rank analysis.

UCP4C in the intestine is necessary for per mutant longevity. To determine if mitochondrial uncoupling is circadian-regulated in the intestine, we first measured mRNA levels of Ucp $4 C$ in dissected whole intestines during the day and night (Fig. 5a). In control intestines, $U c p 4 C$ was low during the day and elevated at night, while $p e r^{01}$ mutants exhibited constitutively high Ucp $4 C$ expression during both day and night. To further evaluate circadian regulation of uncoupling activity in the gut, we assessed mitochondrial membrane potential at different times of day in dissected intestines from wild-type flies and per ${ }^{01}$ mutants, stained with the dye TMRE, which accumulates in mitochondria with higher membrane potential. Loss of membrane potential indicates increased mitochondrial uncoupling. In controls, intestinal mitochondrial membrane potential was indeed circadian-regulated, with higher membrane potential (lower uncoupling) during the day and lower membrane potential (higher uncoupling) at night (Fig. 5b, c). Consistent with loss of circadian regulation, per ${ }^{01}$ mutants exhibited low mitochondrial membrane potential at both timepoints, suggesting high levels of mitochondrial uncoupling during both day and night. This phenotype is not due to changes in mitochondrial abundance, as both controls and per $^{01}$ mutants have similar intestinal mitochondrial populations, as measured by mitoGFP fluorescence (Supplementary Fig. 6A, B). Thus, mitochondrial uncoupling in the Drosophila intestine is circadian-regulated.

Because we found that ubiquitous UCP4C expression was necessary and sufficient for lifespan extension (Fig. 3), we next tested whether intestine-specific UCP4C expression is necessary 
a

Whole intestine Ucp 4C/Actin expression

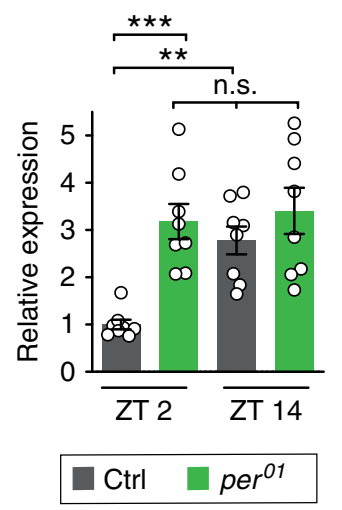

C

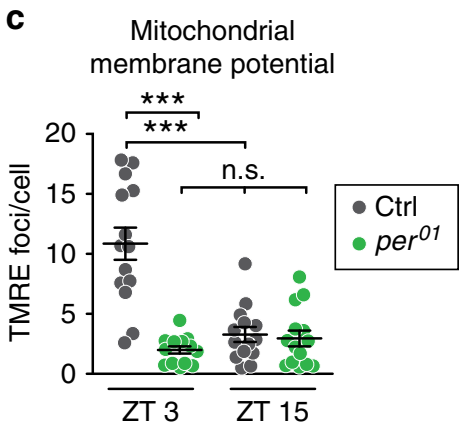

f
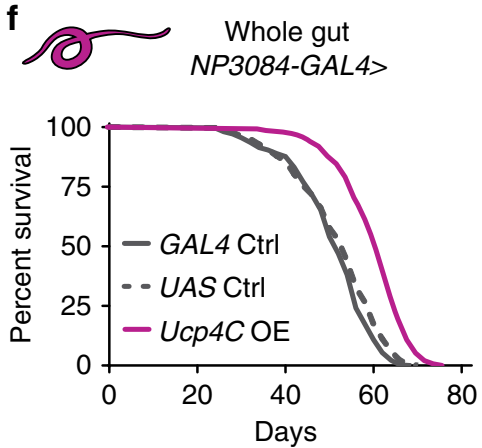

b

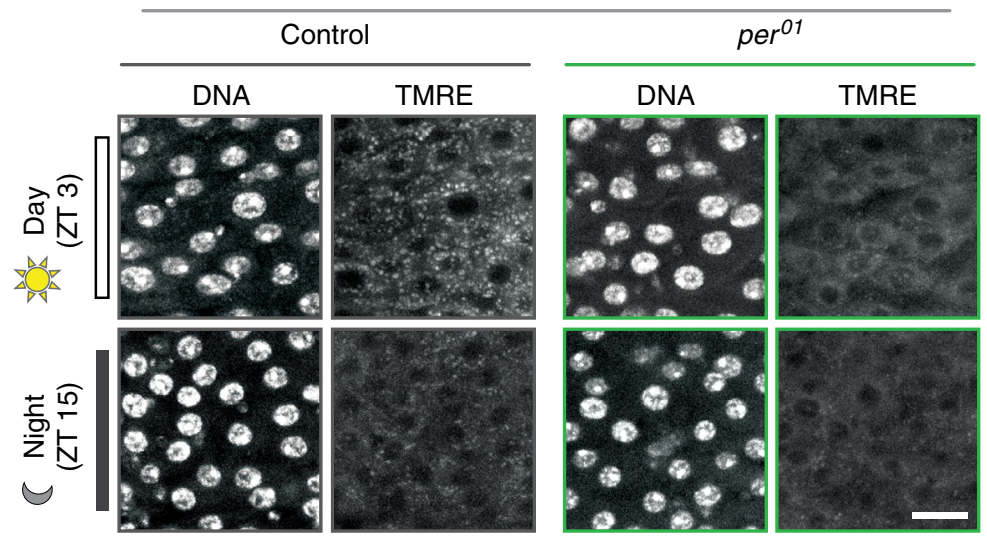

d

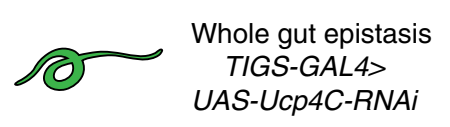

e

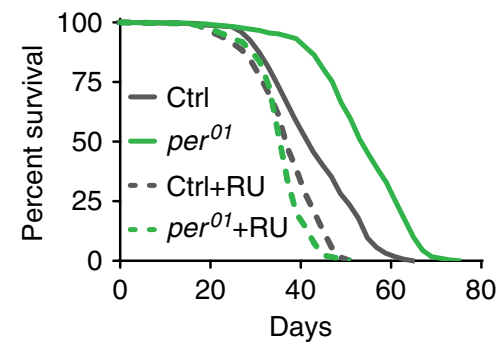

g
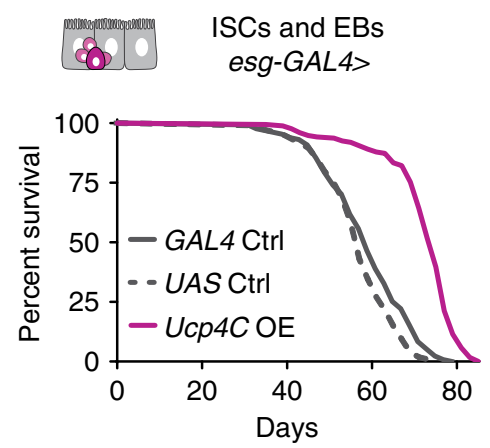
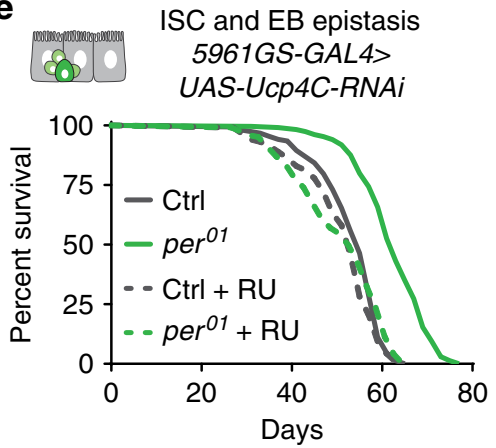

h
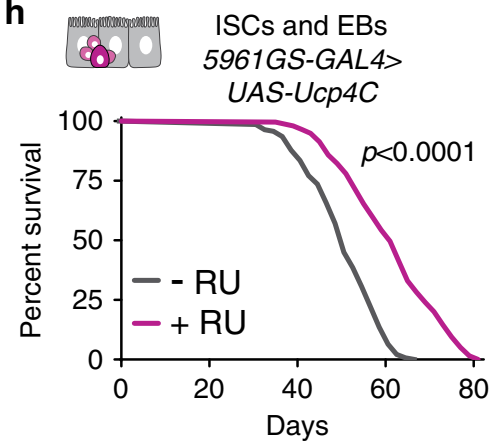

Fig. 5 UCP4C in the intestine is necessary for loss of Per-mediated lifespan extension and sufficient to extend wild-type lifespan. a Whole intestine expression levels of Ucp 4C during day and night show oscillations of Ucp4C expression in controls with constitutively high expression in per01 mutants. b Representative images of the posterior midgut of control (left) and per ${ }^{01}$ mutants (right) stained with Hoechst (DNA) and TMRE during the day and night $(\mathrm{scale} b a r=30 \mu \mathrm{m})$. c While wild-type intestines exhibited high membrane potential during the day and low membrane potential at night, per01 flies exhibited low membrane potential at both times of day. Knockdown of Ucp4C in the whole intestine (d) or ISCs/EBs (e) reverted per01 lifespan to control levels. Relative to controls (gray), flies overexpressing Ucp4C (magenta) in the whole intestine (f) or ISCs and EBs ( $\mathbf{g}$, $\mathbf{h}$ ) had extended lifespan ( $p<0.0001$ for each). See Supplementary Table 1 for $n$ and statistical analysis of lifespans; ${ }^{n . s .} p>0.05,{ }^{\star} p<0.05,{ }^{\star \star} p<0.01,{ }^{\star \star \star} p<0.001 ; p$ values were obtained by ANOVA followed by Tukey's post-hoc test $(\mathbf{a}, \mathbf{c})$ and log-rank analysis (d-h); error bars represent SEM.

and sufficient for lifespan extension. Similar to per rescue, $R N A i$ mediated knockdown of UCP4C in the whole intestine or in ISC/ EB populations reverted per ${ }^{01}$ lifespan to that of controls (Fig. $5 \mathrm{~d}$, e). Moreover, while the overexpression of UCP4C in the nervous system did not alter lifespan (Supplementary Fig. 6C), constitutive expression of UCP4C in the whole intestine (Fig. 5f, Supplementary Fig. 6D) or specifically in ISCs and EBs (Fig. 5g, h) resulted in extended lifespan and lowered mitochondrial membrane potential in otherwise wild-type animals (Supplementary Fig. 6E-H). Thus, intestinal UCP4C expression is not only necessary for the lifespan extension of per ${ }^{11}$ mutants but also sufficient to extend lifespan in otherwise wild-type Drosophila. That is, intestine-specific upregulation of a normally circadianregulated, oscillating physiological function, mitochondrial uncoupling, extends lifespan.

Per and UCP4C control intestinal homeostasis via ROS levels. To understand the underlying mechanism by which mitochondrial uncoupling in the intestine extends lifespan, we tested if loss of per or intestinal UCP4C overexpression delayed aging-related defects in the intestine. When flies age, they typically lose intestinal barrier function, which is also a major predictor of 
a Intestinal barrier dysfunction (45 days)

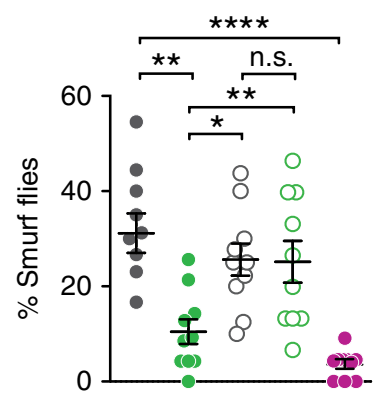

b Mitotic cells (45 days)

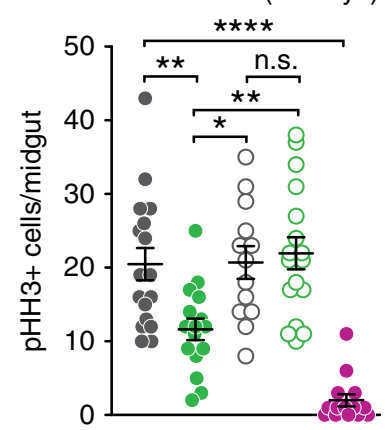

C
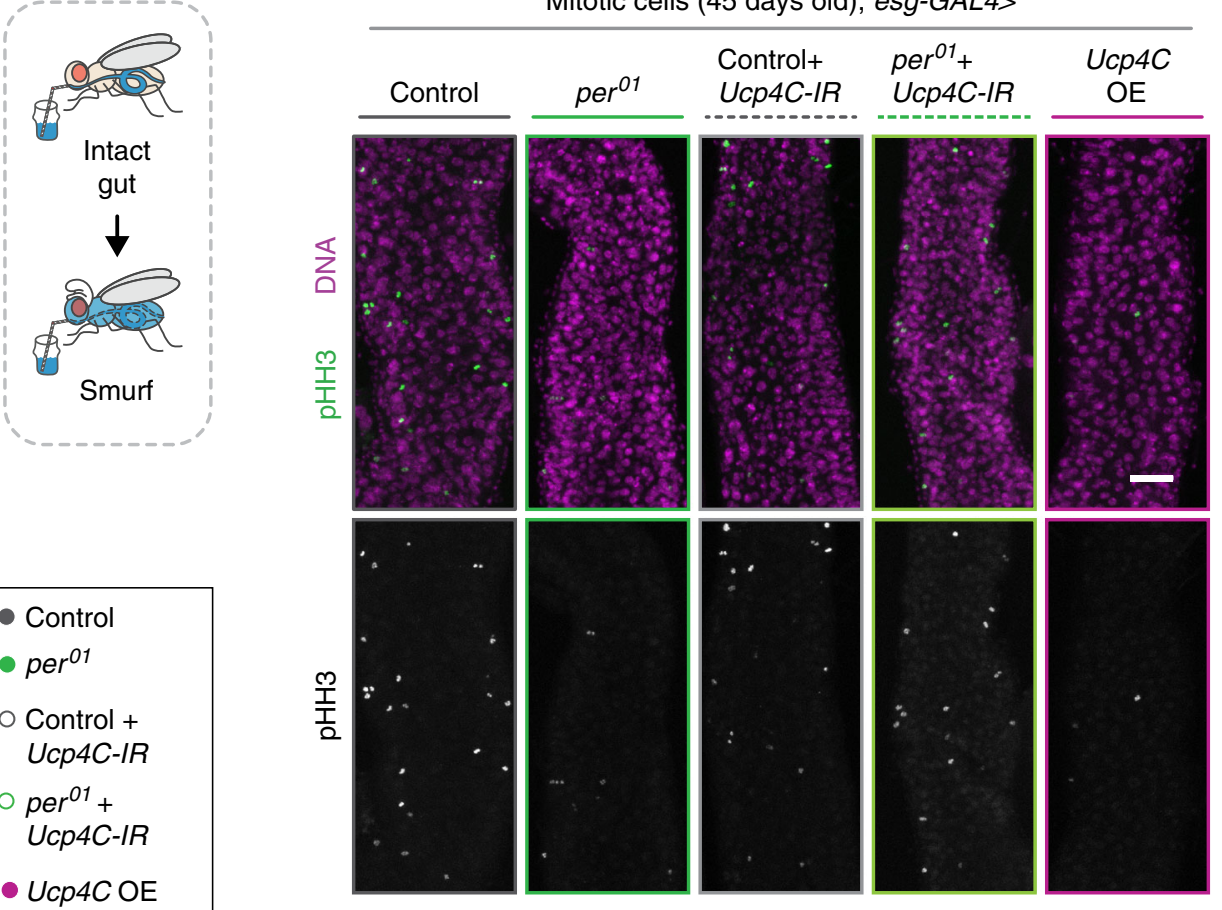

- Control

- per $^{01}$

○ Control +

Ucp4C-IR

$\circ \mathrm{per}^{01}+$

Ucp4C-IR

- Ucp4C OE d

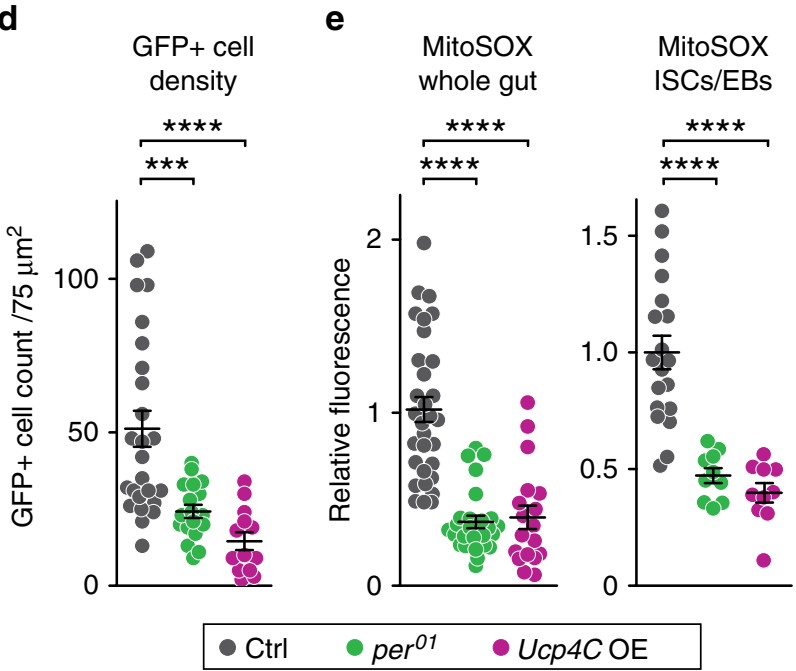

f

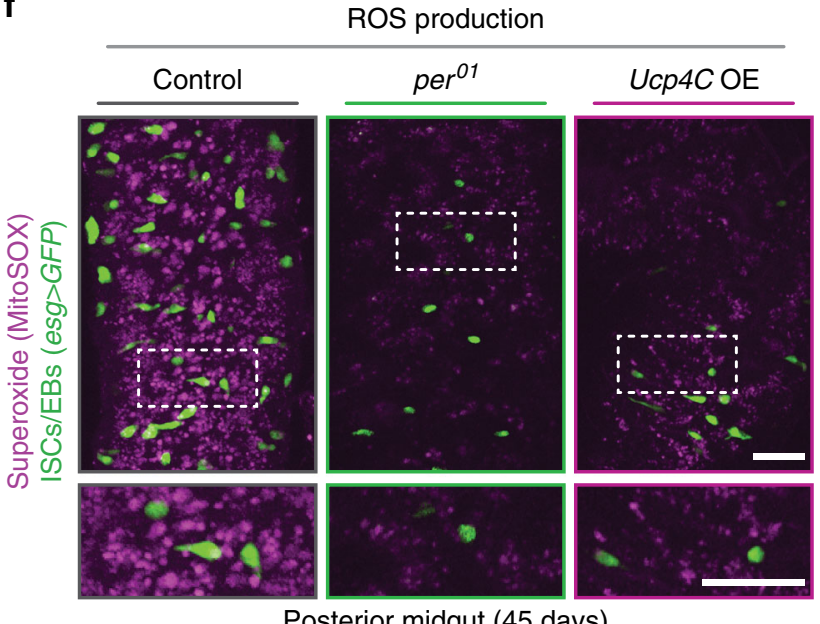

Fig. 6 Loss of period preserves intestinal homeostasis via increased mitochondrial uncoupling and decreased ROS levels. a Smurf assays of 45 -day-old flies showed per ${ }^{01}$ mutants had reduced populations with intestinal barrier dysfunction, a phenotype dependent upon Ucp4C expression, and that overexpression of $U_{c p} 4 \mathrm{C}$ also reduced intestinal barrier dysfunction. b Quantification of intestinal pHH3+ staining showed that per ${ }^{01}$ mutants had lower levels of age-related hyperproliferation dependent on UCP4C expression and that overexpression of UCP4C caused a large reduction in mitotic cells ( $n=$ 13-17 intestines). c Representative images of phospho-histone H3 at residue S10 (pHH3) staining of midguts from 45 -day flies ( $\mathrm{scale}$ bar $=40 \mu \mathrm{m})$. (d, e) per ${ }^{01}$ mutants and Ucp 4C-overexpressing flies exhibited delayed esg + cell overproliferation (e) and lower ROS output of all posterior midgut cells, including ISC/EB populations. f Representative images for MitoSOX staining of aged posterior midguts in control esg-GAL4 > GFP flies, per01; esg-GAL4 > GFP, and esg-GAL4 > GFP;UAS-Ucp4C flies (scale bars $=35 \mu \mathrm{m}$ (top) and $15 \mu \mathrm{m}$ (bottom inset location indicated by dashed lines). See Supplementary Table 1 for $n$ and statistical analysis of lifespans; ${ }^{n . s} . p>0.05,{ }^{\star} p<0.05,{ }^{\star \star} p<0.01,{ }^{\star \star \star} p<0.001,{ }^{\star \star \star \star} p<0.0001 ; p$ values were obtained by ANOVA followed by Tukey's post-hoc test (b, c, e, f); error bars represent SEM.

mortality ${ }^{34-37}$, suggesting that lifespan can be extended by preventing aging-related intestinal barrier dysfunction. We tested for aging-related intestinal barrier dysfunction using the "smurf assay," named after a children's cartoon, which measures leakage of an ingested blue dye $\mathrm{e}^{36,37}$. Consistent with their extended lifespan, old per ${ }^{01}$ mutants showed a lower percentage of "smurfs" relative to controls, indicating a delay in aging-related intestinal barrier dysfunction (Fig. 6a). Moreover, this maintenance of intestinal integrity observed in per $^{01}$ mutants depended on intestinal expression of UCP4C and intestinal overexpression of UCP4C alone in otherwise wild-type flies was sufficient to maintain intestinal integrity. Thus, loss of Per and increased mitochondrial uncoupling in the intestine protect against agingrelated intestinal dysfunction. 
As aging-related intestinal barrier dysfunction is linked to overproliferation and tissue dysplasia in the gut ${ }^{38}$, we next tested if loss of per or intestinal UCP4C overexpression delayed agingrelated cellular proliferation in the gut. Per protein has previously been shown to regulate ISC regeneration during acute intestinal stress $^{39}$. To measure cellular proliferation in the intestines, we stained aged per $^{01}$ and control flies for phospho-histone $\mathrm{H} 3$ at serine 10 ( $\mathrm{pHH} 3)$, a standard proliferative marker that labels mitotic cells (Fig. 6b, c). Further consistent with delayed aging, per $^{01}$ mutants showed fewer $\mathrm{pHH} 3$ positive cells in the gut relative to control flies. To determine if this phenotype depends on mitochondrial uncoupling, we knocked down UCP4C in per ${ }^{01}$ flies and controls. UCP4C knockdown reverted the number of proliferative cells in $p^{01}$ flies to the higher levels seen in controls. Moreover, the overexpression of UCP4C in otherwise wild-type controls was sufficient to decrease age-related overproliferation of ISCs/EBs. Thus, similar to the smurf assay and consistent with lifespan results, intestinal UCP4C expression is both necessary in per $^{01}$ mutants and sufficient in otherwise wildtype controls to suppress aging-related intestinal cellular proliferation.

Because the microbiome and age-related intestinal dysbiosis can contribute to intestinal barrier dysfunction, intestinal dysplasia, and proliferative state $e^{40-42}$, we sought to determine if gut bacteria play a role in period null longevity. First, we measured overall microbial load in aging flies using universal $16 \mathrm{~S}$ bacterial qPCR. Higher bacterial load is associated with aging and mortality. We found that long-lived per $^{01}$ flies did not have decreased bacterial load but instead exhibited an increased bacterial load during aging relative to controls (Supplementary Fig. 7A). To determine if this increased microbial load influenced lifespan, we fed control and per ${ }^{01}$ flies throughout adulthood an antibiotic cocktail that reduced intestinal bacterial levels to nearly undetectable levels (Supplementary Fig. 7B). Feeding antibiotics for the entire lifespan had no impact on the extended longevity of per nulls relative to controls (Supplementary Fig. 7C). Together, these data show that the microbiome present in our animals did not cause per ${ }^{01}$ longevity.

Intestinal dysfunction and increased aging-related cellular proliferation are closely associated with elevated Reactive Oxygen Species (ROS) production. ROS can serve as important mitogenic signals in stem cells and their differentiated progeny cells in the fly ${ }^{43}$; elevated ROS production in the Drosophila intestine is thought to lead to increased misdifferentiation of proliferative cells, ISCs, and $\mathrm{EBs}^{44}$. Because mitochondrial uncoupling can restrict ROS output ${ }^{45-47}$, we hypothesized that uncoupled per $^{01}$ mutants have extended longevity due to decreased mitochondrial ROS production in the intestine, leading to a decrease in agerelated overproliferation of esg-positive (esg+) ISC/EB cells. To test this, we stained the intestines of aged per ${ }^{01}$ mutants, flies expressing UCP4C constitutively in ISCs and EBs, and controls with MitoSOX Red, a fluorescent mitochondrial ROS (superoxide) indicator (Fig. 6f). Both per ${ }^{01}$ mutants and intestinal UCP4C-expressing flies exhibited reduced esg + populations and decreased mitochondrial superoxide production relative to controls in the whole posterior midgut, as well as in ISC/EB populations (Fig. 6d, e). Together, these results suggest that loss of Per function and increased mitochondrial uncoupling via upregulated UCP4C decrease ROS levels in the intestine.

Uncoupling drugs extend lifespan via gut homeostasis. To extend our genetic experiments, we tested if pharmacological induction of mitochondrial uncoupling recapitulates the intestinal phenotypes of per ${ }^{1}$ mutants and extends lifespan. We tested low dietary concentrations of two compounds, the mitochondrial uncoupling compound 2,4-DNP and uncoupling-agent/antioxidant beta-hydroxytoluene (BHT). To identify the effective dose of these compounds, we first showed that both compounds extended the lifespan of wild-type male flies when used within a range of physiological concentrations (Fig. 7a-c, Supplementary Fig. $6 \mathrm{~A})^{48,49}$. To ensure that these drugs did not cause diet restriction, we confirmed that DNP or BHT did not decrease feeding rate (Supplementary Fig. 6B). We found that these drugs did not extend the lifespan of per ${ }^{01}$ mutants, suggesting that these mutants are already receiving the maximal benefit of mitochondrial uncoupling (Supplementary Fig. 6C, D). Consistent with the hypothesis that uncoupling-induced lifespan extension is mediated by reduced cellular proliferation and decreased ROS levels, dietary DNP at concentrations that extend lifespan also reduce age-related esg + cell overproliferation and mitochondrial ROS output (Fig. 7d, e). Thus, pharmacological induction of mitochondrial uncoupling extends lifespan and recapitulates many of the metabolic phenotypes of per $^{01}$ mutants and UCP4Cexpressing flies.

To test whether the mitochondrial uncoupling-induced suppression of cellular overproliferation is specific to agingrelated cellular overproliferation and misdifferentiation or may act as a general mechanism to suppress cellular overproliferation, we tested the effects of the uncoupling drug DNP on an induced intestinal tumor model. Notch-Delta signaling normally keeps proliferative intestinal cells (ISCs/EBs) at a homeostatic level of differentiation and division ${ }^{50}$; loss of Notch in ISCs and EBs causes cellular overproliferation and the formation of ISC-derived intestinal tumors ${ }^{50,51}$. Changes to ISCs that alter differentiation and result in overproliferation are a common step in cancer development ${ }^{52}$. To test if mitochondrial uncoupling could slow tumor progression in the gut, we induced ISC tumors by RNAi suppression of Notch signaling, followed by feeding of 2,4-DNP (to induce mitochondrial uncoupling) or vehicle. Dietary 2,4DNP slowed the progression of ISC tumors (Fig. 7f, g) and extended the short lifespan of these animals (Fig. 7h). These results suggest that pharmacological induction of mitochondrial uncoupling can metabolically suppress stem cell proliferation not only during normal aging but also during tumorigenesis.

\section{Discussion}

Circadian regulators are known to control the organism's metabolism, including mitochondrial uncoupling;, ${ }^{2,53}$ the relationship between circadian regulation, metabolism, and lifespan is highly complex. Here we showed that the male circadian mutant per ${ }^{01}$ is long-lived due to a mechanism independent of, and additive with, canonical methods of life extension. Loss of period increased mitochondrial uncoupling, resulting in hyperphagic yet lean animals that maintain intestinal health with age. Ablating circadian regulation via loss of Per or increasing circadian-regulated mitochondrial uncoupling specifically in the intestine was sufficient to lower intestinal ROS levels, preserve proliferative homeostasis and gut barrier function, and extend lifespan. These beneficial intestinal phenotypes were recapitulated in wild-type flies pharmacologically by feeding low doses of two different mitochondrial uncoupling drugs. Finally, 2,4-DNP feeding was also capable of delaying tumor formation in a model of genetically induced ISC overproliferation. Thus, while mitochondrial uncoupling has previously been proposed to extend lifespan and healthspan, we demonstrate here an underlying mechanism related to intestinal tissue homeostasis $45,49,54,55$. Our overarching hypothesis posits that loss of Per and circadian rhythms leads to a state of increased mitochondrial uncoupling, which decreases ROS production in the intestine (Supplementary Fig. 9). This prevents aging-related stem cell overproliferation and 
a

Whole lifespan

2,4 DNP feeding

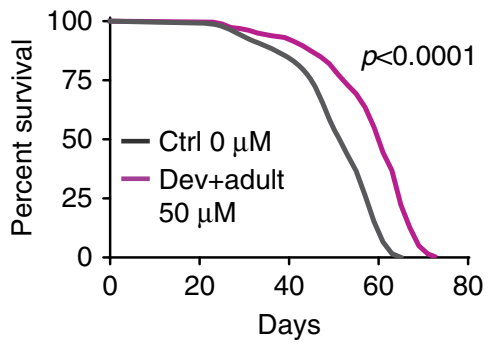

d
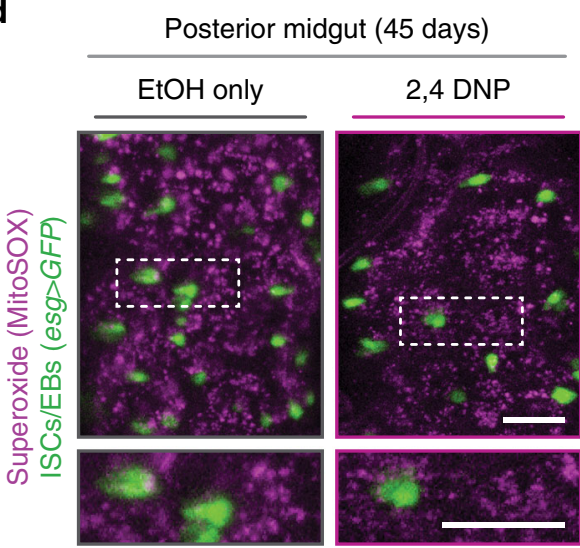

f esg-GAL4 $4^{\text {ts }}>$ UAS-GFP; UAS-Notch-RNAi
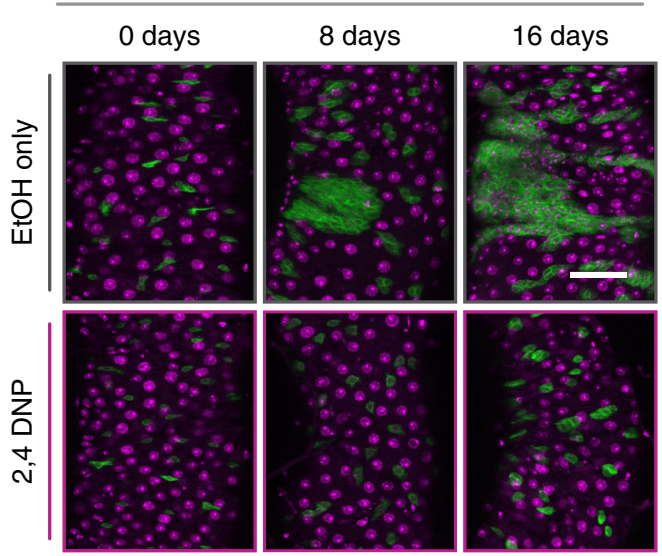

esg $>$ GFP DNA b

Adulthood

2,4 DNP feeding

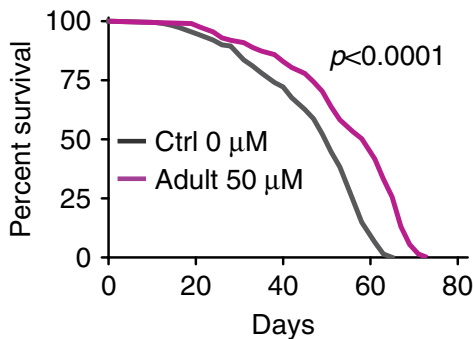

e

e
density

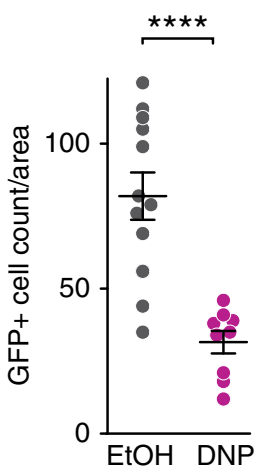

g

$\%$ GFP + signal of posterior midgut area

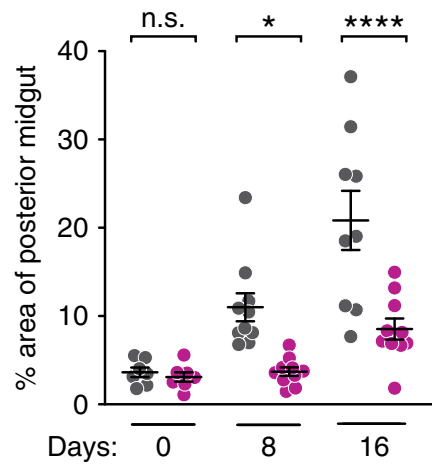

C
Median lifespan

Adult 2,4 DNP feeding

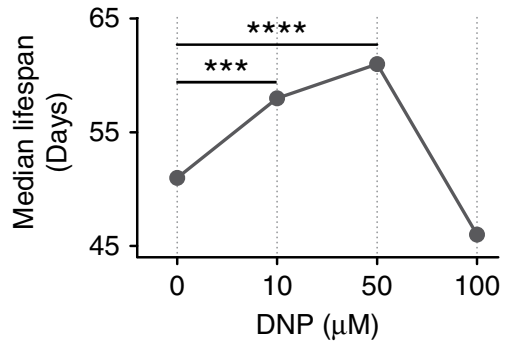

Mitosox

whole gut

Mitosox

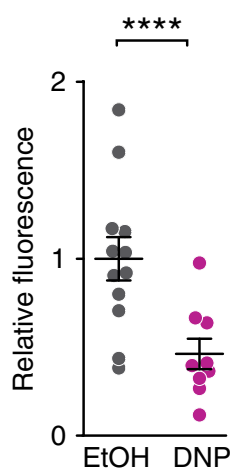

ISCs/EBs

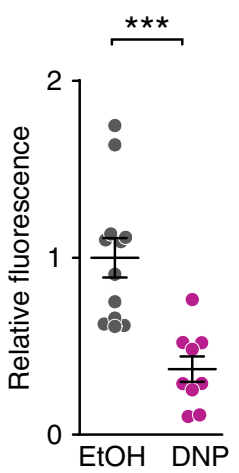

h

esg-GAL4 ${ }^{\text {ts }}>U A S-N o t c h-R N A i$ lifespan $30^{\circ} \mathrm{C}$
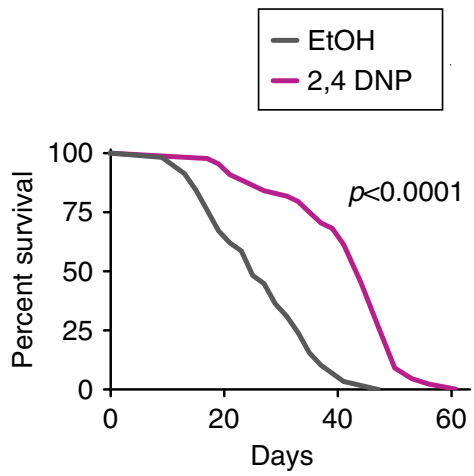

Fig. 7 Pharmacological reduction of ROS via uncoupling preserves intestinal homeostasis and extends lifespan. Flies fed the mitochondrial uncoupler 2,4-DNP (magenta) either throughout their lifespan (a) or only during adulthood (b) showed extended lifespan relative to vehicle controls (gray, $p<$ 0.0001 for each). c 2,4-DNP feeding increased median lifespan in a dose-dependent manner. $\mathbf{d}$ Representative images of MitoSOX staining of posterior midguts in esg-GAL4 > GFP flies fed vehicle or DNP (scale bars $=25 \mu \mathrm{m}$, top; $10 \mu \mathrm{m}$, bottom, inset location indicated by dashed lines). e Flies fed 2,4-DNP exhibited: fewer esg + cells and thus lower ISC/EB overproliferation with age (left); decreased Mitosox staining of the entire posterior midgut and thus decreased mitochondrial superoxide output (middle); and decreased MitoSOX staining specifically in ISCs/EBs marked by GFP (right). f Representative images of midguts from flies exhibiting loss of Notch-mediated tumor formation (scale bar $=35 \mu \mathrm{m}$ ). Relative to vehicle-fed flies, flies with Notch-induced tumor formation that were fed 2,4-DNP exhibited delayed tumor formation as measured by GFP + area in the midgut $(\mathbf{g})$ and extended lifespans (h, $p<$ 0.0001 for each). See Supplementary Table 1 for $n$ and statistical analysis of lifespans; ${ }^{n . s .} p>0.05,{ }^{\star} p<0.05,{ }^{\star \star} p<0.01,{ }^{\star \star \star} p<0.001,{ }^{\star \star \star \star *} p<0.0001$; $p$ values were obtained by log-rank analysis $(\mathbf{a}, \mathbf{b}, \mathbf{c}, \mathbf{h})$, unpaired two-tailed t-test (e), and ANOVA followed by Tukey's post-hoc test ( $(\mathbf{g})$; error bars represent SEM.

misdifferentiation, resulting in lifespan extension and, in the case of genetically induced tumors in Drosophila, inhibition of tumor growth.

Our initial finding that loss of Per extends the lifespan of Drosophila is controversial, as other groups reported that per null mutants have shorter lifespan than controls ${ }^{5,8,9}$. We observed this short lifespan phenotype only with per males fed very high protein content diets. Environmental factors such as diet composition were strictly controlled in our experiments. Furthermore, we confirmed our findings using novel CRISPR techniques to delete 
period from the whole organism and specifically in the intestine (Fig. 4), resulting in robust lifespan extension. Contradictions in the field have been attributed in part to differences in pathogen status or the microbiome ${ }^{10,12}$. Upon microbiome removal via antibiotic feeding, we still observed significant lifespan extension in period nulls, indicating that the microbiome found in our flies does not contribute to this phenotype (Supplementary Fig 7). Regional and diet-based differences in the microbiome can significantly alter lifespan of the organisms ${ }^{56}$. Our experiments do not rule out a more pathogenic microbiome in some environmental conditions that could preferentially shorten period mutant lifespan. Consistent with this, per mutants display varying susceptibility and resistance to pathogenic infection $16,19,57,58$. In Drosophila, the composition of the microbiome can greatly influence the inflammation level (including ROS production) and the resulting proliferative status of the intestine $e^{59,60}$. Further investigation into the intestinal microbiome composition of circadian mutants and resulting inflammation and immune response or other environmental factors could shed light onto these findings.

The molecular mechanism underlying circadian regulation of mitochondrial UCP expression remains unknown. Consistent with previous findings that circadian regulators such as PER2 and REVERBa can regulate the expression of uncoupling protein UCP1 in mice 61,62 , we showed that the expression of mitochondrial uncoupling proteins $\mathrm{UCP} 4 \mathrm{C} / \mathrm{B}$ is circadian-regulated in wild-type Drosophila and constitutively high in period nulls (Fig. 3). However, there are no canonical Clock-binding E-box sequences present in the promoter regions of these genes, suggesting that Clock and Cycle do not directly regulate UCP4C/B expression. Thus, we predict that Per disruption causes the accumulation of an unidentified transcription factor that directly regulates UCP expression; this will be an area of active future investigation. Moreover, UCPs are activated posttranslationally by specific fatty acid components and lipid metabolism is altered in per mutants ${ }^{8}$. Additional understanding of the circadian regulation of UCP expression and activity could support the benefits of metabolic treatments such as intermittent fasting or different treatment schedules for antidiabetic drugs such as metformin, as well as other therapeutics that regulate metabolism and lifespan.

Our main hypothesis posits that mild mitochondrial uncoupling mitigates ROS production in aged mitochondria, which is consistent with previous reports ${ }^{45,46}$. This mild reduction in proton gradient may trade energy inefficiency with the benefit of reduced ROS output. While the precise mechanism by which a mildly reduced proton gradient mitigates ROS production remains unclear, it is known that high ROS production is correlated with increased membrane potential ${ }^{46,47}$. UCPs could serve to mitigate this potentially damaging state throughout the circadian cycle as energy demands change with time of day.

This UCP-lowered ROS production in the intestine is sufficient to maintain proliferative homeostasis in the gut (Fig. 6). Our data also support the hypothesis that ROS or ROS-induced signals sensed by ISCs/EBs cause their proliferation and contribute to dysregulated tissue homeostasis in the gut. We found that UCP4C overexpression in ISCs/EBs alone is sufficient to lower ROS output of the whole posterior midgut, including fully differentiated enterocyte cells (ECs), not only the ISC/EB population from which ECs are derived. Future work will investigate how this UCP-mediated lowered ROS production is passed on from ISCs/ EBs to differentiated daughter ECs, the major cell type of the intestine. While it is possible that ECs inherit this low ROS metabolic state via UCPs in EB mitochondria, this phenotype could also be due to non-cell-autonomous signaling from ISCs/ EBs to surrounding ECs. The mechanism(s) by which ISC/EBs pass on this metabolic state via uncoupling may be a diffusible signal, such as mitochondrially generated ROS itself, metabolic rewiring due to altered metabolite utilization, or a new type of "mitokine" that is currently undiscovered. Further investigation into how mitogenic signals are coordinated between cell-types of the intestine may reveal the mechanism by which this metabolic state is maintained. In addition, tight regulation of mitochondrial metabolism and oxidative state is conserved in the process of mammalian ISC differentiation ${ }^{63,64}$. Thus, our work supports a role for oxidative stress in the maintenance of ISC identity with age, a role likely to be evolutionarily conserved.

Our use of mitochondrial uncoupling compounds such as 2,4DNP showed that they can recapitulate lifespan extension in Drosophila. 2,4-DNP was originally used in high doses in the 1930s as weight loss supplements or diet pills ${ }^{65}$, but dangerous side-effects such as hyperthermia, cataracts, and cardiac dysfunction led to its discontinuation. More recent findings in mice ${ }^{49,66}$ suggest that very low doses can reduce body fat and potentially increase lifespan, but the tissue-specific mechanism by which this compound acts to increase longevity remained elusive. Here we provide evidence that 2,4-DNP limits ROS output of the intestine, thus preserving intestinal homeostasis with advanced age and even delaying tumor growth (Fig. 7). Together, these data suggest that mitochondrial uncoupling induces a metabolic state that inhibits cellular proliferation. In yeast, mitochondrial uncoupling is known to signal to the nucleus and cause profound changes in gene expression ${ }^{67}$; future investigation will determine if this is true for multicellular organisms and if these changes in gene expression cell-autonomously inhibit proliferation of stem cells. Our data provide insight into circadian-regulated mitochondrial function to improve intestinal healthspan and organismal longevity and implicate mitochondrial uncoupling as a potential therapeutic to control stem cell overproliferation/misdifferentiation and delay aging.

\section{Methods}

Fly strains. cycle $\left(c y c^{01}\right)$ period $\left(\right.$ per $\left.{ }^{01}\right)$ and timeless $\left(\right.$ tim $\left.^{01}\right)$ mutants outcrossed to the Canton-S (CS) background as controls were obtained from Jaga Giebultowicz. $p B A C-U c p 4 C^{\mathrm{e} 03988}, U A S-D N-S 6 K$ (6911) were obtained from the Bloomington Stock Center. UAS-Ucp4A-RNAi, UAS-Ucp4B-RNAi, and UAS-Ucp4C-RNAi were obtained from the Vienna Drosophila Resource Center. UAS-Ucp4C was received from flyORF. UAS-GFP-Atg8 from Eric Baehrecke, ubi-GAL4 from Marc Dionne, esg-GAL4, NP3084-GAL4, daughterless-Gene-Switch (daGS), elav-Gene-Switch and TIGS-2 (TIGS-GAL4), 5961GS-GAL4, dilp2-GAL4 and UAS-Reaper from David W. Walker, UAS-per10 and UAS-per24, and UAS-DN-Clock from Amita Sehgal, esg ${ }^{\text {ts }}$ GAL4, and UAS-Notch-RNAi were from Ben Ohlstein. All experiments with multiple transgenes used flies that have undergone 12 generations of out-crossing into a $w^{1118} C S$ control and/or per $01, w^{1118} C S$ mutant background.

UAS-CRISPR line construction. Multiple gRNAs targeting per, or acp $98 A B$ were constructed according to (Port and Bullock, Nature Methods, 2016). In short, pCFD6 (Addgene \#73915) was digested with BbsI-HF (NEB \#R3539S) and the linearized plasmid gel purified. For each construct, inserts were generated in three separate PCR reactions using $p C F D 6$ as the template and the primers listed below. The resulting three inserts and the pCFD6 backbone were then assembled by NEBuilder HiFi DNA Assembly (NEB \#E2621L) for each construct. Each construct was then micro-injected at integration site $\mathrm{Su}(\mathrm{Hw})$ attP5 (Bestgene Inc.) and Sanger sequenced.

UAS-per-gRNA, guide RNA targets:

1. GCTTTTCTACACACACCCGG

2. CACGTGCGATATGATCCCGG

3. GGAGTCCACACACAACACCA

4. TACTCGTCCATAGACCACGC

per_PCR1fwd-GCGGCCCGGGTTCGATTCCCGGCCGATGCAGCTTTTCTA CACACACCCGGGTTTTAGAGCTAGAAATAGCAAG

per_PCR1rev-CCGGGATCATATCGCACGTGTGCACCAGCCGGGAATCG AACCC

per_PCR2 $f w d$-CACGTGCGATATGATCCCGGGTTTTAGAGCTAGAAATA GCAAG

per_PCR2rev-TGGTGTTGTGTGTGGACTCCTGCACCAGCCGGGAATCG AACCC

per_PCR3fwd-GGAGTCCACACACAACACCAGTTTTAGAGCTAGA AATAGCAAG 
per_PCR3rev-

ATTTTAACTTGCTATTTCTAGCTCTAAAACGCGTGGTCTAT

GGACGAGT

ATGCACCAGCCGGGAATCGAACCC

UAS-Ctrl-gRNA, guide RNA targets:

1. GTGTCCCCTTATTCGTGCGG

2. CACACTATCAAAGGATGACG

3. ATAAGGGGACACACTATCAA

4. AGTGTGTCCCCTTATTCGTG

acp 98AB PCR1fwd-

GCGGCCCGGGTTCGATTCCCGGCCGATGCAGTGTCCCCTTAT

TCGTGCGGGTTTTAGAGCTAGAAATAGCAAG

acp98AB PCR1rev-CGTCATCCTTTGATAGTGTGTGCACCAG

CCGGGAATCGAACCC

$a c p 98 A B$ PCR2fwd-CACACTATCAAAGGATGACGGTTTTAGAGCTAG

AAATAGCAAG

acp98AB_PCR2rev-TTGATAGTGTGTCCCCTTATTGCACCAGCCGGGAA

TCGAACCC

acp98AB_PCR3fwd-ATAAGGGGACACACTATCAAGTTTTAGAGCTAGA

AATAGCAAG

acp98AB_PCR3rev-ATTTTAACTTGCTATTTCTAGCTCTAAAACCACG

AATAAGGGGA

CACACTTGCACCAGCCGGGAATCGAACCC

Fly media. Drosophila were reared from embryos in low-density bottles with standard medium containing $3.8 \%$ glucose, $1.9 \%$ sucrose, $3 \%$ yeast nutritional flake (Lab Scientific), 6.5\% cornmeal, 0.8\% agar, supplemented with 1.5\% methylparaben mix ( $10 \%$ methylparaben in ethanol), and $1 \%$ propionic acid. Adult flies that eclosed within a $24 \mathrm{~h}$ period were collected and transferred to "adult medium" containing $3.8 \%$ glucose, $1.9 \%$ sucrose, $8 \%$ cornmeal, $1 \%$ agar, and either $0.01,0.5$, 3,5 , or $10 \%$ YE (Difco) supplemented with $1.5 \%$ methylparaben mix and $1 \%$ propionic acid for lifespan and biochemical analysis. All percentages given in $\mathrm{wt} / \mathrm{v}$ except methylparaben mix, propionic acid given in $\mathrm{v} / \mathrm{v}$.

Drug supplementation in media for lifespan. All drugs were supplemented into cooled $\left(65^{\circ} \mathrm{C}\right)$ liquid adult medium (containing $3 \%$ YE) following preparation to the following final concentration(s): RU486 (Cayman Chemical) was dissolved in ethanol was supplemented into medium after eclosion at a final concentration of $100 \mu \mathrm{g} / \mathrm{mL}$ (with the exception of $d a G S>U A S-U c p 4 c$ and controls, $5 \mu \mathrm{g} / \mathrm{mL}$ ) vehicle controls were supplemented with same volume of ethanol alone. Developmental induction was achieved by adding RU 486 at concentration of $5 \mu \mathrm{g} / \mathrm{mL}$, or ethanol vehicle, followed by adults being aged on standard food containing no RU486. For microbiota clearance medium contained $500 \mu \mathrm{g} / \mathrm{ml}$ ampicillin, $50 \mu \mathrm{g} / \mathrm{ml}$ tetracycline, and $200 \mu \mathrm{g} / \mathrm{ml}$ rifamycin in $50 \%$ ethanol. Same volume of $50 \%$ ethanol alone was used as the vehicle control. For uncoupler feeding 2,4-DNP or BHT purchased from Sigma was dissolved in ethanol fresh at the time of making food and added into the media, at the indicated concentrations. 2,4-DNP (0.01-0.1 mM) BHT (0.1-1 mM), the same volume of ethanol alone was used as vehicle control.

Lifespan analysis and starvation. Newly eclosed flies $(\sim 24 \mathrm{~h})$ were collected and allowed to mate for $48 \mathrm{~h}$. Female and male flies were separated and maintained at a density of 30-35 flies per vial in a humidified, temperature-controlled $\left(25^{\circ} \mathrm{C}\right)$ incubator with a 12-h light-dark (LD) cycle. For starvation analysis, flies were aged for 7 days in vials containing indicated diets and then transferred into $1 \%$ agar in a humidified, temperature-controlled incubator with 12 -h LD cycle at $25^{\circ} \mathrm{C}$. Death was scored every 1-2 days, with live flies transferred into fresh vials every 2-3 days. Statistical significance was determined by log-rank analysis.

Western blot analysis. Whole-body lysates of 10-day-old male flies (30 flies/ sample/timepoint) were separated by SDS-PAGE using standard procedures. Membranes were probed with antibodies against AMPK phospho-T184 at 1:1000 (Cell Signaling, 40H9); anti-phospho-S6K T398 (Cell Signaling, 9209), anti-GFP (Cell Signaling, D5.1), antiphospho AKT s505 (Cell Signaling, 4054), and pan AKT at 1:1000 (Cell Signaling, 9272); and horseradish peroxidase (HRP)-conjugated monoclonal mouse antiactin antibody at 1:5000 (Sigma-Aldrich, A3854). Rabbit antibodies were detected using HRP-conjugated anti-rabbit IgG antibodies at 1:2000 (Cell Signaling, 7074). Mouse antibodies were detected using HRPconjugated anti-mouse IgG antibodies 1:2000 dilution (Cell Signaling, 7076). ECL chemiluminescence reagent (Pierce) was used to visualize horseradish peroxidase activity and detected by CCD camera using a Kodak Image Station. A minimum of four independent samples of each condition were used for statistical analysis and quantification.

Quantitative real-time PCR. Thirty whole male flies, or ten whole intestines per biological replicate were used for total RNA extraction via TRIzol reagent (Invitrogen) following manufacturer protocols. Samples were treated with DNase, and then cDNA was synthesized by the Revertaid First Strand cDNA Synthesis Kit (Thermo Scientific). A minimum of four independent samples were used for statistical analysis and quantification. For 16s bacterial rDNA quantification 10 whole flies (washed twice in 70\% ethanol, and twice in PBS) were used for total DNA extraction via the Power Soil DNA isolation kit (Mo Bio). Universal primers for the $16 \mathrm{~S}$ ribosomal RNA gene were against variable regions 1 (V1F) and $2(\mathrm{~V} 2 \mathrm{R})$, as previously published ${ }^{68}$.

Equalized amplicons of Actin5C were used as a reference gene to normalize expression via the following primer sets:

Ucp4A-fwd- TTTGACTACGCGGACTCATTC

Ucp4A-rev- CGCGGTATTGCATATTGGACTT

$U c p 4 B$-fwd- AACACAGTCTTTAGGCCAGCA

Ucp4B-rev- CCGTGAGGTAGAGTTCAACCG

Ucp4C-fwd- ACAAACGTCGCTGATCCACTA

Ucp4C-rev- GGAAGACACACGACTCGGC

period-fwd- GGTTGCTACGTCCTTCTGGA

period-rev- TGTGCCTCCTCCGATATCTT

16Sr-fwd- AGAGTTTGATCCTGGCTCAG

16Sr-rev- CTGCTGCCTYCCGTA

Actin5C-fwd- TTGTCTGGGCAAGAGGATCAG

Actin5C-rev- ACCACTCGCACTTGCACTTTC

Cold shock recovery assay. Seven to ten days old males were anesthetized on ice and individually placed in vials and loaded into a DAM5 activity monitor. Flies were maintained at $4{ }^{\circ} \mathrm{C}$ for $1 \mathrm{~h}$, and then placed in a $25^{\circ} \mathrm{C}$ incubator and allowed to recover for $4 \mathrm{~h}$. Individual flies were scored as recovered at the time three beam breaks were recorded by the activity monitor system. A minimum of 22 flies per condition were used for statistical analysis (log-rank analysis).

Quantification of triglycerides levels. Lipids were extracted from five whole flies in a chloroform:ethanol solution (2:1 vol/vol), and nonpolar lipids (fatty acid, triacylglycerol) were separated by thin-layer chromatography with an n-hexane/ diethylether/glacial acetic acid solution $(70: 30: 1, \mathrm{vol} / \mathrm{vol} / \mathrm{vol})$. Plates were air-dried and stained (with $0.2 \%$ Amido Black $10 \mathrm{~B}$ in $1 \mathrm{M} \mathrm{NaCl}$ ), and lipid bands were quantified by photo densitometry using ImageJ software. Densitometry values were normalized to the mass of the homogenized flies used in the sample following a reference standard of pre-measured coconut oil or butter. A minimum of six independent samples were used for statistical analysis and quantification.

Intestinal barrier dysfunction assay. The "smurf fly"/intestinal barrier dysfunction assay was performed similarly to ref. ${ }^{36}$. Flies were aged on regular medium until the day of the smurf assay. Dyed medium was prepared by the addition of FD\&C Blue No. 1 at a final concentration of $2.5 \% \mathrm{wt} / \mathrm{vol}$. A fly was counted as a smurf when dye coloration was observed outside the digestive tract. Comparisons of smurf proportion per timepoint were carried out using binomial tests to calculate the probability of having as many smurfs in population A as in population B, as well as ANOVA for proportions of smurf flies per replicate vial with a minimum of 7 vials of 15-31 flies per replicate.

Feeding assay. Analysis of capillary feeding ("the CAFE assay") was performed similarly to ref. ${ }^{69}$ with minor modifications. Briefly, ten flies were placed in vials with wet tissue paper as a water source and a capillary food source ( $3.8 \%$ glucose $1.9 \%$ sucrose, $3 \%$ YE, and $0.2 \%$ FD\&C Blue No. 1). Feeding was monitored for at least $24 \mathrm{~h}$, replacing depleted capillaries as necessary. A minimum of eight groups of ten flies per condition were used for each experiment.

$\mathrm{CO}_{2}$ respiration assay. Measurement of $\mathrm{CO}_{2}$ production was performed similarly to ref. ${ }^{70}$. Briefly, ten flies were placed in a sealed pipette tip-capillary respirometer (without anesthesia) containing a small amount of soda lime. Respirometers were placed capillary side down in $\mathrm{dH}_{2} \mathrm{O}$ containing $0.1 \%$ blue food dye in a sealed TLC chamber placed in a $25^{\circ} \mathrm{C}$ incubator. Empty sealed respirometers (containing no flies) were used to subtract blue dye background migration from experimental (flycontaining) samples. Flies were tested for $2 \mathrm{~h}$ between at circadian timepoints, and $\mathrm{CO}_{2}$ production rate was indirectly determined by the amount of liquid movement up the capillary per hour after background subtraction. At least six groups of ten flies were used per genotype for quantification for each condition. For mitochondrial drug feeding experiments, flies were placed on food containing the following compounds for $24 \mathrm{~h}: 500 \mu \mathrm{M}$ Oligomycin, $1 \mathrm{mM}$ rotenone, $5 \mathrm{mM}$ 2,4-DNP, $0.5 \%$ stearate, or ethanol vehicle controls.

Mitochondrial purification for downstream assays. Fifty whole flies were gently crushed in chilled mitochondrial isolation medium (MIM) $(250 \mathrm{mM}$ sucrose, 10 $\mathrm{mM}$ Tris- $\left.\mathrm{HCl}(\mathrm{pH} 7.4), 0.15 \mathrm{mM} \mathrm{MgCl}_{2}\right)$ passed through a $40 \mu \mathrm{m}$ filter basket and spun twice at $1000 \times g$ for $5 \mathrm{~min}$ at $4{ }^{\circ} \mathrm{C}$ to remove nuclei and debris. The supernatant was then spun at $4000 \times \mathrm{g}$, for $10 \mathrm{~min}$ at $4{ }^{\circ} \mathrm{C}$. The pellet, containing the mitochondria, was washed once in fresh MIM buffer and then total protein content of the resulting pellet was determined by the Bradford Assay.

Mitochondrial oxygen consumption measurements. Protein content of purified mitochondria was normalized, and samples were resuspended in modified MiR05 buffer (0.5 mM EGTA, $3 \mathrm{mM} \mathrm{MgCl}_{2}, 60 \mathrm{mM}$ K-lactobionate, $20 \mathrm{mM}$ taurine, 
$10 \mathrm{mM} \mathrm{KH}_{2} \mathrm{PO}_{4}, 20 \mathrm{mM}$ HEPES, $110 \mathrm{mM}$ sucrose, and $0.25 \mathrm{~g} / \mathrm{L}$ BSA, $\mathrm{pH}$ 7.2). Mitochondrial respiration was measured at $25^{\circ} \mathrm{C}$ using high-resolution respirometry (Oxygraph-2k, Oroboros). To determine max ADP stimulated respiration initiated through complexes I and II, the following sequential injections were made (final concentration in chamber): $5 \mathrm{mM}$ glutamate plus $5 \mathrm{mM}$ malate, $5 \mathrm{mM}$ succinate, $1 \mathrm{mM}$ ADP. To determine nonphosphorylating leak respiration, $3 \mu \mathrm{M}$ Oligomycin was then added. To measure reserve electron transport capacity of the mitochondria, carbonyl cyanide m-chlorophenyl hydrazine (CCCP) $0.5 \mathrm{mM}$ stock was titrated into the chamber at $1 \mu \mathrm{L}$ increments. Residual nonmitochondrial oxygen consumption was determined by the addition of $1 \mu \mathrm{M}$ rotenone, and 2.5 $\mu \mathrm{M}$ antimycin A. Respiration rates of mitochondria initiated through complexes I, II, and IV were determined by the following injection/reaction schema (final concentrations in chamber): $5 \mathrm{mM}$ glutamate plus $5 \mathrm{mM}$ malate, $1 \mathrm{mM}$ ADP, 0.5 $\mu \mathrm{M}$ rotenone, $5 \mathrm{mM}$ succinate, $2.5 \mu \mathrm{M}$ antimycin $\mathrm{A}$, and $1 \mathrm{mM}$ ascorbate plus 0.25 $\mathrm{mM} \mathrm{N}$-tetramethyl-1,4-phenylenediamine, $0.5 \mathrm{mM} \mathrm{KCN}$. A minimum of four independent mitochondrial preps were run for each condition. For determination of palmitate stimulated respiration and inhibition by GTP mitochondria were washed three times in modified MiR05 containing $1 \%$ fatty acid free BSA to remove any free fatty acids present. Respiration was stimulated through complex II by the addition of $5 \mathrm{mM}$ succinate in the presence of $0.5 \mu \mathrm{M}$ rotenone. BSA-conjugated to palmitate (Agilent) was added to a final Palmitate concentration of $10 \mathrm{nM}$, followed by $2 \mathrm{mM}$ GTP (Thermo), and finally $2.5 \mu \mathrm{M}$ antimycin A. A minimum of three independent mitochondrial preps were run for each condition.

Membrane potential JC-1 assay. Protein content of purified mitochondria was normalized, and samples were resuspended at a concentration of $200 \mu \mathrm{g} / \mathrm{mL}$ in modified MiR05 buffer $(0.5 \mathrm{mM}$ EGTA, $3 \mathrm{mM} \mathrm{MgCl}$, $60 \mathrm{mM}$ K-lactobionate, 20 $\mathrm{mM}$ taurine, $10 \mathrm{mM} \mathrm{KH}_{2} \mathrm{PO}_{4}, 20 \mathrm{mM}$ HEPES, $110 \mathrm{mM}$ sucrose, and $0.25 \mathrm{~g} / \mathrm{L} \mathrm{BSA}$, pH 7.2) containing $20 \mu \mathrm{g} / \mathrm{mL} \mathrm{JC}-1$ dye. $(488 \mathrm{ex} / 530 \mathrm{em})$ and $(533 \mathrm{ex} / 590 \mathrm{em})$ spectra were monitored via plate reader at $25^{\circ} \mathrm{C}$. To measure membrane potential at steady-state ATP production stimulated through complexes I and II: $5 \mathrm{mM}$ glutamate plus $5 \mathrm{mM}$ malate, $5 \mathrm{mM}$ succinate, $1 \mathrm{mM}$ ADP were added to the preparation. To determine membrane potential upon inhibited ATP synthesis, $3 \mu \mathrm{M}$ oligomycin was then added. To further control for the function of the dye, $25 \mu \mathrm{M}$ of CCCP was then added to dissipate the proton gradient. A minimum of four independent mitochondrial preps were run for statistical analysis.

Blue NativePAGE and in-gel activity assay. Assays were performed as in ref. ${ }^{71}$. Mitochondria were purified from ten thoraces of 7-10-day-old male flies and BNPAGE was performed using NativePAGE gels from Life Technologies, following the manufacturer's instructions. Mitochondria were suspended in NativePAGE sample buffer (Life Technologies) supplemented with $1 \%$ digitonin and protease inhibitors, incubated on ice for $20 \mathrm{~min}$ and centrifuged at $20,000 \times \mathrm{g}$ for $30 \mathrm{~min}$ at $4{ }^{\circ} \mathrm{C}$, the supernatant was recovered, G-250 (Life Technologies) sample additive and NativePAGE Sample Buffer was added before loading onto 3-12\% precast Bis-Tris NativePAGE gels (Life Technologies). Electrophoreses was performed using the NativePAGE Running buffer (as anode buffer, from Life technologies) and the NativePAGE Running buffer containing 0.4\% Coomassie G-250 (cathode buffer). Protein complexes were revealed by staining with Novex Colloidal Blue staining kit (Life Technologies). Complex I activity in native gels was performed by incubating gels in $0.1 \mathrm{mg} / \mathrm{mL} \mathrm{NADH}, 2.5 \mathrm{mg} / \mathrm{mL}$ nitrotetrazolium blue chloride, $5 \mathrm{mM}$ Tris- $\mathrm{HCl}$ ( $\mathrm{pH}$ 7.4) overnight at room temperature. Gels were imaged using a BioRad station and densitometry was performed in ImageJ.

Circadian locomotor activity. Post eclosion, flies were reared in a humidified, temperature-controlled incubator with 12 -h $\mathrm{LD}$ cycle at $25^{\circ} \mathrm{C}$. Seven-day-old adults were anesthetized under light $\mathrm{CO}_{2}$ and placed individually into Drosophila Activity Monitor (DAM) tubes, containing standard food. Flies were monitored using a DAM2 system (Trikinetics) for 3 days in 12-h LD cycle for circadian entrainment, followed by 6 days of constant darkness (DD) to assess free running locomotor activity. ClockLab (Coulbourn Industries) was used to determine the rhythmicity and period of the fly populations in constant darkness. A minimum of 16 flies per genotype were used for each experiment.

TMRE/MitoSOX intestinal staining. Flies were anesthetized on ice and intestines were dissected in cold Schneider's Medium (Thermo Fisher Scientific). Intestines were then incubated in $75 \mathrm{nM}$ of TMRE (Thermo Fisher Scientific) in Schneider's Medium, for $10 \mathrm{~min}$ at room temperature. Samples were rinsed three times for $30 \mathrm{~s}$ with a solution consisting of $20 \mathrm{nM}$ of TMRE Schneider's Medium. For MitoSOX staining dissected intestines were immersed in $50 \mu \mathrm{M}$ MitoSOX Red (Invitrogen) in Schneider's Medium for $5 \mathrm{~min}$ at room temperature, and then washed three times for $30 \mathrm{~s}$ in Schneider's Medium. MitoSOX or TMRE samples were quickly mounted in Schneider's Medium containing $1.5 \mu \mathrm{g} / \mathrm{mL}$ Hoechst stain. Intestines were imaged within 20 min on a Ziess LSM800 confocal microscope. $Z$-stacks spanning the entire posterior midgut were taken. The quantification of TMRE or MitoSOX was performed using ImageJ in which mean fluorescent intensity values were quantified. TMRE foci were quantified using the Image $J^{72}$ local maxima tool, using identical thresholding for all images. Foci number were normalized to the number of nuclei in the measured field of view. The quantification of MitoSOX output of ISCs/EBs was determined by mean intensity from individual $Z$-planes with regions of interest defined by esgGFP expression, cell size, and basal location within the intestinal epithelium. Mean intensity of MitoSOX fluorescence levels in all ISCs/EBs were averaged for each individual intestine. A minimum of nine midguts were used for each genotype or drug treatment.

Phospho-histone H3 immunostaining. Briefly, flies were anesthetized on ice and intestines were dissected in cold PBS. Samples were then fixed in PBS $+0.1 \%$ Triton X-100 containing $4 \%$ paraformaldehyde at room temperature for $30 \mathrm{~min}$ and rinsed three times in PBS $+0.1 \%$ Triton X-100 for $10 \mathrm{~min}$ at room temperature. Blocking was performed in 5\% BSA in PBS $+0.1 \%$ triton X-100 for $1 \mathrm{~h}$ at room temperature. Primary antibody, anti-phospho-histone H3 (S10) (Cell Signaling, 9701), was added 1:250 in 5\% BSA in PBS $+0.1 \%$ triton X-100 and incubated overnight at $4{ }^{\circ} \mathrm{C}$ rotating. After washing three times in PBS $+0.1 \%$ Triton X-100 secondary antibody, anti-rabbit AlexaFluor-488 (Invitrogen) was added $1: 250$, and $1.5 \mu \mathrm{g} / \mathrm{mL}$ Hoechst stain (Thermo) in $5 \%$ BSA in PBS $+0.2 \%$ triton X100 and incubated overnight at $4{ }^{\circ} \mathrm{C}$ rotating. After washing, intestines were then mounted in Vectashield mounting medium (Vector Labs) and Imaged using Zeiss Ziess LSM800. pHH3 positive cells were quantified using the Image ${ }^{72}$ local maxima tool, with identical thresholding for all images. $\mathrm{pHH} 3$ numbers were normalized to the area of the posterior midgut imaged. A minimum of ten intestines were used for each quantification.

esg $^{\text {ts }}>$ UAS-Notch-RNAi intestine staining. esg $^{t s}>U A S-N o t c h-R N A i$ flies were reared at $18{ }^{\circ} \mathrm{C}$. Upon eclosion flies were collected, sexed, and placed on media containing $0.05 \mathrm{mM} 2,4-\mathrm{DNP}$, or ethanol vehicle food at $30^{\circ} \mathrm{C}$. Timepoints of 0 8 , and 16 days were taken. Intestines were dissected, fixed, and stained as above pHH3 protocol, with anti-GFP (Cell Signaling, D5.1) and $1.5 \mu \mathrm{g} / \mathrm{mL}$ Hoechst stain. The quantification of GFP area was performed by using the ImageJ count particle tool in the GFP channel normalized to total area of the posterior midgut that was imaged. Seven to thirteen guts were used for each condition and timepoint.

Statistical analysis. Prism7 (GraphPad) was used to perform the statistical analysis significance is expressed as $p$ values $\left({ }^{n} p p>0.05,{ }^{*} p<0.05,{ }^{* *} p<0.01\right.$, ${ }^{* * *} p<$ 0.001 ). For two group comparisons, unpaired, two-tailed $t$-test was used, when data met criteria for parametric analysis (normal distribution and similar variance). Mann-Whitney $U$-test was used in case of nonparametric analysis. For more than two group's comparison, ANOVA with Bonferroni or Tukey's post-hoc test was performed. Kruskal-Wallis with dunn's post-hoc for data that was not distributed appropriately for the parametric comparisons. For comparison of survival curves, Log-rank (Mantel-Cox) test was used.

Reporting summary. Further information on research design is available in the Nature Research Reporting Summary linked to this article.

\section{Data availability}

The authors declare that all data supporting the findings of this study are available within the paper and its Supplementary Information files. The data for all graphs shown in this paper are available in source data file. Additional raw data, and replicate experiments will be made available upon reasonable request.

Received: 8 February 2019; Accepted: 11 March 2020; Published online: 21 April 2020

\section{References}

1. Panda, S. Circadian physiology of metabolism. Science 354, 1008-1015 (2016)

2. Brown, S. A. Circadian metabolism: from mechanisms to metabolomics and medicine. Trends Endocrinol. Metab. 27, 415-426 (2016).

3. Dubrovsky, Y. V., Samsa, W. E. \& Kondratov, R. V. Deficiency of circadian protein CLOCK reduces lifespan and increases age-related cataract development in mice. Aging 2, 936-944 (2010).

4. Kondratov, R. V., Kondratova, A. A., Gorbacheva, V. Y., Vykhovanets, O. V. \& Antoch, M. P. Early aging and age-related pathologies in mice deficient in BMAL1, the core componentof the circadian clock. Genes Dev. 20, 1868-1873 (2006)

5. Vaccaro, A., Issa, A. R., Seugnet, L., Birman, S. \& Klarsfeld, A. Drosophila clock is required in brain pacemaker neurons to prevent premature locomotor aging independently of its circadian function. PLoS Genet. 13, e1006507 (2017).

6. Hendricks, J. C. et al. Gender dimorphism in the role of cycle (BMAL1) in rest, rest regulation, and longevity in Drosophila melanogaster. J. Biol. Rhythms 18, $12-25$ (2003) 
7. Means, J. C. et al. Drosophila spaghetti and doubletime link the circadian clock and light to caspases, apoptosis and tauopathy. PLoS Genet. 11, e1005171 (2015).

8. Katewa, S. D. et al. Peripheral circadian clocks mediate dietary restrictiondependent changes in lifespan and fat metabolism in Drosophila. Cell Metab. 23, 143-154 (2016).

9. Krishnan, N., Kretzschmar, D., Rakshit, K., Chow, E. \& Giebultowicz, J. M. The circadian clock gene period extends healthspan in aging Drosophila melanogaster. Aging 1, 937-948 (2009).

10. Ulgherait, M. et al. Dietary restriction extends the lifespan of circadian mutants tim and per. Cell Metab. 24, 763-764 (2016).

11. Park, N., Cheon, S., Son, G. H., Cho, S. \& Kim, K. Chronic circadian disturbance by a shortened light-dark cycle increases mortality. Neurobiol. Aging 33, 1122 el111-1122 e1122 (2012).

12. Wang, J. et al. Per2 induction limits lymphoid-biased haematopoietic stem cells and lymphopoiesis in the context of DNA damage and ageing. Nat. Cell Biol. 18, 480-490 (2016).

13. Fu, L., Pelicano, H., Liu, J., Huang, P. \& Lee, C. C. The circadian gene Period2 plays an important role in tumor suppression and DNA damage response in vivo. Cell 111, 41-50 (2002).

14. Lee, S., Donehower, L. A., Herron, A. J., Moore, D. D. \& Fu, L. Disrupting circadian homeostasis of sympathetic signaling promotes tumor development in mice. PLoS ONE 5, el0995 (2010).

15. Shirasu-Hiza, M. M., Dionne, M. S., Pham, L. N., Ayres, J. S. \& Schneider, D. S. Interactions between circadian rhythm and immunity in Drosophila melanogaster. Curr. Biol. 17, R353-R355 (2007).

16. Stone, E. F. et al. The circadian clock protein timeless regulates phagocytosis of bacteria in Drosophila. PLoS Pathog. 8, el002445 (2012)

17. So, A. Y.-L., Bernal, T. U., Pillsbury, M. L., Yamamoto, K. R. \& Feldman, B. J. Glucocorticoid regulation of the circadian clock modulates glucose homeostasis. Proc. Natl Acad. Sci. USA 106, 17582-17587 (2009).

18. Antoch, M. P., Toshkov, I., Kuropatwinski, K. K. \& Jackson, M. Deficiency in PER proteins has no effect on the rate of spontaneous and radiation-induced carcinogenesis. Cell Cycle 12, 3673-3680 (2013).

19. Allen, V. W. et al. Period-regulated feeding behavior and TOR signaling modulate survival of infection. Curr. Biol. 26, 184-194 (2016).

20. Grimaldi, B. et al. PER2 controls lipid metabolism by direct regulation of PPAR $\gamma$. Cell Metab. 12, 509-520 (2010).

21. Tanoue, S., Krishnan, P., Krishnan, B., Dryer, S. E. \& Hardin, P. E. Circadian clocks in antennal neurons are necessary and sufficient for olfaction rhythms in Drosophila. Curr. Biol. 14, 638-649 (2004).

22. Brand, A. H. \& Perrimon, N. Targeted gene expression as a means of altering cell fates and generating dominant phenotypes. Development 118, 401-415 (1993).

23. Kapahi, P. et al. Regulation of lifespan in Drosophila by modulation of genes in the TOR signaling pathway. Curr. Biol. 14, 885-890 (2004).

24. Broughton, S. J. et al. Longer lifespan, altered metabolism, and stress resistance in Drosophila from ablation of cells making insulin-like ligands. Proc. Natl Acad. Sci. USA 102, 3105-3110 (2005).

25. Da-Re, C. et al. UCP4C mediates uncoupled respiration in larvae of Drosophila melanogaster. EMBO Rep. 15, 586-591 (2014).

26. Hanak, P. \& Jezek, P. Mitochondrial uncoupling proteins and phylogenesis-UCP4 as the ancestral uncoupling protein. FEBS Lett. 495, 137-141 (2001).

27. Azzu, V. \& Brand, M. D. The on-off switches of the mitochondrial uncoupling proteins. Trends Biochem. Sci. 35, 298-307 (2010).

28. Woyda-Ploszczyca, A. M. \& Jarmuszkiewicz, W. Different effects of guanine nucleotides (GDP and GTP) on protein-mediated mitochondrial proton leak. PLoS ONE 9, e98969 (2014).

29. Woyda-Ploszczyca, A. M. \& Jarmuszkiewicz, W. The conserved regulation of mitochondrial uncoupling proteins: from unicellular eukaryotes to mammals. Biochim. Biophys. Acta 1858, 21-33 (2017).

30. Allada, R. \& Chung, B. Y. Circadian organization of behavior and physiology in Drosophila. Annu Rev. Physiol. 72, 605-624 (2010).

31. Port, F. \& Bullock, S. L. Augmenting CRISPR applications in Drosophila with tRNA-flanked Cas9 and Cpf1 sgRNAs. Nat. Methods 13, 852-854 (2016).

32. Delventhal, R. et al. Dissection of central clock function in Drosophila through cell-specific CRISPR-mediated clock gene disruption. eLife 8, e48308 (2019).

33. Mueller, J. L. et al. Cross-species comparison of Drosophila male accessory gland protein genes. Genetics 171, 131-143 (2005).

34. Biteau, B. et al. Lifespan extension by preserving proliferative homeostasis in Drosophila. PLoS Genet. 6, e1001159 (2010).

35. Wang, L., Karpac, J. \& Jasper, H. Promoting longevity by maintaining metabolic and proliferative homeostasis. J. Exp. Biol. 217, 109-118 (2014).

36. Rera, M. et al. Modulation of longevity and tissue homeostasis by the Drosophila PGC-1 homolog. Cell Metab. 14, 623-634 (2011).

37. Rera, M., Clark, R. I. \& Walker, D. W. Intestinal barrier dysfunction links metabolic and inflammatory markers of aging to death in Drosophila. Proc. Natl Acad. Sci. USA 109, 21528-21533 (2012).
38. Hu, D. J. \& Jasper, H. Epithelia: understanding the cell biology of intestinal barrier dysfunction. Curr. Biol. 27, R185-R187 (2017).

39. Karpowicz, P., Zhang, Y., Hogenesch, J. B., Emery, P. \& Perrimon, N. The circadian clock gates the intestinal stem cell regenerative state. Cell Rep. 3, 996-1004 (2013).

40. Akagi, K. et al. Dietary restriction improves intestinal cellular fitness to enhance gut barrier function and lifespan in D. melanogaster. PLoS Genet. 14, e1007777 (2018).

41. Clark, RebeccaI. et al. Distinct shifts in microbiota composition during Drosophila aging impair intestinal function and drive mortality. Cell Rep. 12, 1656-1667 (2015)

42. Guo, L., Karpac, J., Tran, Susan, L. \& Jasper, H. PGRP-SC2 promotes gut immune homeostasis to limit commensal dysbiosis and extend lifespan. Cell 156, 109-122 (2014).

43. Owusu-Ansah, E. \& Banerjee, U. Reactive oxygen species prime Drosophila haematopoietic progenitors for differentiation. Nature 461, 537-541 (2009).

44. Hochmuth, C. E., Biteau, B., Bohmann, D. \& Jasper, H. Redox regulation by Keap1 and Nrf2 controls intestinal stem cell proliferation in Drosophila. Cell Stem Cell 8, 188-199 (2011).

45. Fridell, Y. W., Sanchez-Blanco, A., Silvia, B. A. \& Helfand, S. L. Targeted expression of the human uncoupling protein 2 (hUCP2) to adult neurons extends life span in the fly. Cell Metab. 1, 145-152 (2005).

46. Mailloux, R. J. \& Harper, M. E. Uncoupling proteins and the control of mitochondrial reactive oxygen species production. Free Radic. Biol. Med. 51, 1106-1115 (2011).

47. Korshunov, S. S., Skulachev, V. P. \& Starkov, A. A. High protonic potential actuates a mechanism of production of reactive oxygen species in mitochondria. FEBS Lett. 416, 15-18 (1997).

48. Lou, P. H. et al. Mitochondrial uncouplers with an extraordinary dynamic range. Biochem. J. 407, 129-140 (2007).

49. Caldeira da Silva, C. C., Cerqueira, F. M., Barbosa, L. F., Medeiros, M. H. \& Kowaltowski, A. J. Mild mitochondrial uncoupling in mice affects energy metabolism, redox balance and longevity. Aging Cell 7, 552-560 (2008).

50. Ohlstein, B. \& Spradling, A. Multipotent Drosophila intestinal stem cells specify daughter cell fates by differential notch signaling. Science 315, 988-992 (2007).

51. Biteau, B., Hochmuth, C. E. \& Jasper, H. JNK activity in somatic stem cells causes loss of tissue homeostasis in the aging Drosophila gut. Cell Stem Cell 3, 442-455 (2008).

52. Zhai, Z. et al. Accumulation of differentiating intestinal stem cell progenies drives tumorigenesis. Nat. Commun. 6, 10219 https://www.nature.com/ articles/ncomms10219\#supplementary-information (2015)

53. Giebultowicz, J. M. Circadian regulation of metabolism and healthspan in Drosophila. Free Radic. Biol. Med. https://doi.org/10.1016/j. freeradbiomed.2017.12.025 (2017).

54. Barros, M. H., Bandy, B., Tahara, E. B. \& Kowaltowski, A. J. Higher respiratory activity decreases mitochondrial reactive oxygen release and increases life span in Saccharomyces cerevisiae. J. Biol. Chem. 279, 49883-49888 (2004).

55. Padalko, V. I. Uncoupler of oxidative phosphorylation prolongs the lifespan of Drosophila. Biochemistry 70, 986-989 (2005).

56. Douglas, A. E. Contradictory results in microbiome science exemplified by recent Drosophila research. mBio, 9, https://doi.org/10.1128/mBio.01758-18 (2018).

57. Shirasu-Hiza, M. M., Dionne, M. S., Pham, L. N., Ayres, J. S. \& Schneider, D. S. Interactions between circadian rhythm and immunity in Drosophila melanogaster. Curr. Biol. 17, R353-R355 (2007).

58. Liu, J. et al. The circadian clock Period 2 gene regulates gamma interferon production of NK cells in host response to lipopolysaccharide-induced endotoxic shock. Infect. Immun. 74, 4750-4756 (2006).

59. Buchon, N., Broderick, N. A., Chakrabarti, S. \& Lemaitre, B. Invasive and indigenous microbiota impact intestinal stem cell activity through multiple pathways in Drosophila. Genes Dev. 23, 2333-2344 (2009).

60. Lee, K.-A. et al. Bacterial-derived uracil as a modulator of mucosal immunity and gut-microbe homeostasis in Drosophila. Cell 153, 797-811 (2013).

61. Gerhart-Hines, Z. et al. The nuclear receptor Rev-erba controls circadian thermogenic plasticity. Nature 503, 410-413 (2013).

62. Fu, L., Patel, M. S., Bradley, A., Wagner, E. F. \& Karsenty, G. The molecular clock mediates leptin-regulated bone formation. Cell 122, 803-815 (2005).

63. Berger, E. et al. Mitochondrial function controls intestinal epithelial stemness and proliferation. Nat. Commun. 7, 13171 https://www.nature.com/articles/ ncomms13171\#supplementary-information (2016).

64. Myant, KevinB. et al. ROS production and NF- $\mathrm{kB}$ activation triggered by RAC1 facilitate WNT-driven intestinal stem cell proliferation and colorectal cancer initiation. Cell Stem Cell 12, 761-773 (2013).

65. Grundlingh, J., Dargan, P. I., El-Zanfaly, M. \& Wood, D. M. 2,4-dinitrophenol (DNP): a weight loss agent with significant acute toxicity and risk of death. J. Med. Toxicol. 7, 205-212 (2011). 
66. Goldgof, M. et al. The chemical uncoupler 2,4-dinitrophenol (DNP) protects against diet-induced obesity and improves energy homeostasis in mice at thermoneutrality. J. Biol. Chem. 289, 19341-19350 (2014).

67. Epstein, C. B. et al. Genome-wide responses to mitochondrial dysfunction. Mol. Biol. Cell 12, 297-308 (2001).

68. Claesson, M. J. et al. Comparison of two next-generation sequencing technologies for resolving highly complex microbiota composition using tandem variable 16S rRNA gene regions. Nucleic Acids Res. 38, e200-e200 (2010).

69. Ja, W. W. et al. Prandiology of Drosophila and the CAFE assay. Proc. Natl Acad. Sci. USA 104, 8253-8256 (2007).

70. Yatsenko, A. S., Marrone, A. K., Kucherenko, M. M. \& Shcherbata, H. R. Measurement of metabolic rate in Drosophila using respirometry. J. Vis. Exp. e51681, https://doi.org/10.3791/51681 (2014).

71. Garcia, C. J., Khajeh, J., Coulanges, E., Chen, E. I. \& Owusu-Ansah, E. Regulation of mitochondrial complex I biogenesis in Drosophila flight muscles. Cell Rep. 20, 264-278 (2017).

72. Schneider, C. A., Rasband, W. S. \& Eliceiri, K. W. NIH Image to ImageJ: 25 years of image analysis. Nat. Methods 9, 671-675 (2012).

\section{Acknowledgements}

We thank all Shirasu-Hiza and Canman lab members for support, productive discussions, and feedback on this manuscript. We also thank David W. Walker, Amita Sehgal, Ben Ohlstein, and Jaga Giebultowicz for fly lines. Stocks obtained from the Bloomington Drosophila Stock Center (NIH P40OD018537) were used in this study. This work was supported by: NIH training grant 5T32DK007328 (M.U.), the Charles H. Revson Foundation (M.U.), AFAR Glenn Foundation Postdoctoral Fellowship for Aging Research (M. U.), Columbia's Summer Undergraduate Research Fellowship (A.C., S.F.M., M.O.), Cardiovascular Translational Research Training Grant (5T32HL120826) (R.D.), F31GM125363 (C.J.G.), NIH R01GM117407 (J.C.C.); NIH R35GM119793 (M.P.); Hirschl Foundation (E.O-A. and M.S-H.); NIH R35GM124717 (E.O-A.); NIH R21DK112074 (E. O-A.); NIH R35GM127049 (M.S-H.); and NIH R01AG045842 (M.S-H.).

\section{Author contributions}

M.U. and M.S-H. conceived of the experiments. Experiments were performed and analyzed by: M.U. (all experiments); A.C., S.F.M., R.D., C.R.W., Y.R. (lifespan experiments); H.X.K. performed cloning of CRISPR constructs and analysis of lifespan data; C.J.G. (mitochondrial complex formation and in-gel assays). R.D., C.R.W., J.C.C., M.P., E.O-A., M.O. made intellectual and technical contributions. J.C.C., M.U., and M.S-H. designed the figures and wrote the manuscript.

\section{Competing interests}

The authors declare no competing interests.

\section{Additional information}

Supplementary information is available for this paper at https://doi.org/10.1038/s41467 020-15617-x.

Correspondence and requests for materials should be addressed to M.S.-H.

Peer review information Nature Communications thanks the anonymous reviewer(s) for their contribution to the peer review of this work.

Reprints and permission information is available at http://www.nature.com/reprints

Publisher's note Springer Nature remains neutral with regard to jurisdictional claims in published maps and institutional affiliations.

Open Access This article is licensed under a Creative Commons Attribution 4.0 International License, which permits use, sharing, adaptation, distribution and reproduction in any medium or format, as long as you give appropriate credit to the original author(s) and the source, provide a link to the Creative Commons license, and indicate if changes were made. The images or other third party material in this article are included in the article's Creative Commons license, unless indicated otherwise in a credit line to the material. If material is not included in the article's Creative Commons license and your intended use is not permitted by statutory regulation or exceeds the permitted use, you will need to obtain permission directly from the copyright holder. To view a copy of this license, visit http://creativecommons.org/ licenses/by/4.0/.

(C) The Author(s) 2020 